Original Research Paper

\title{
Marketing your Business School on Social Media: Analyzing Social Media Content for Adopting the Right Strategies
}

\author{
${ }^{1}$ Vikram Kumar and ${ }^{1}$ Ramakrishnan Raman \\ ${ }^{1,2}$ Symbiosis International (Deemed Univrsity) and Symbiosis Institue of Business Management, Pune, India
}

Article history

Received: 23-04-2019

Revised: 19-08-2019

Accepted: 04-09-2019

Corresponding Author:

Vikram Kumar

Symbiosis International

(Deemed University) and

Symbiosis Institue of Business

Management, Pune, India

Email: vikram@srvmedia.com

\begin{abstract}
Many Indian Business Schools and higher educational institutions have realized the importance of social media and the interactions in the social media platforms for branding their institution. Higher education institutions, specifically Indian Business Schools (B Schools) have been using the social media as a platform to connect to the prospective students, employers and alumni. With growing competition it has become imperative for Indian B Schools to use social media and communicate with all stake holders. For many Indian B Schools updating and maintaining the social media channels has become one of the most important digital marketing activity. Statista Research reveals the India takes the first place with 300 million users on Facebook and in the second position with 210 million Facebook users is the United States of America. Hence Indian B schools use Facebook as one of their important social media platform to build their identity and also to connect and communicate with prospective aspirants. In this paper the authors have taken posts from Facebook of renowned Indian B Schools. The format used and the topic of the posts have been taken from the Facebook pages of top 100 Indian B Schools. The B school were selected by considering National Institutional Ranking Framework (NIRF) developed by Ministry of Human Resource Development Government of India-India Rankings 2018 under Management category. The total posts that have been taken for the study is 19612. All the posts that were posted on the Facebook from May 2018 to January 2019 were included. The authors have analyzed the content to find the topics and format that increases the engagement and those that do not. This paper provides some strategies on how Indian B Schools can run their social media campaigns, with specific reference to Facebook posts, to better market their offering and also to increase organic reach of the posts.
\end{abstract}

Keywords: Social Media, Marketing B School, Facebook, Digital Marketing, Strategy, Digital Campaign

\section{Introduction}

Creating a brand image and building brand equity is of vital importance for any B School. Brand building can help to attract best students to seek admission to the management programs offered by the school. In the last two decades, India has seen a phenomenal growth of business education. Building a brand image has become imperative for Indian B Schools and this has also become one of the important agenda for the administration and leadership. Intense challenge from local and global management Institutions, obliterated global boundaries, has made B schools to not only take conventional print and television to reach out to the aspirants informing about the achievements, but also use the new age social media to inform and engage with the aspirants. Social media has been a subject of discussion and debate. Several authors have 
studies the effects of social media on human behavior (Barker, 2009; Kolbitsch and Maurer, 2006). Social media and its use in the educational environment has also been studies by several researchers (Augustsson, 2010; Kabilan et al., 2010). Social media as a marketing tool has been extensively researched by several researchers (Constantinides and Fountain, 2008; Ghauri et al., 2003; Kim et al., 2010; Mangold and Faulds, 2009; Spaulding, 2010).

B Schools conventionally used snail mail, brochures and also campus visits to attract students. (Hayes et al., 2009). But with the advent of internet and social media, students are actively using social media to reach out to the B Schools (Hayes et al., 2009). According to a Pew Research Center report (Pew Research Center, 2019) $62 \%$ (median value) of adults in the emerging economies are users of Facebook as their preferred Social Networking Sites (SNS). This is also driving B Schools to use social media as a tool and especially Facebook to engage with the prospective students (Sandvig, 2016). Using social media as a marketing tool by higher education institutions is an alluring proposition, due to the positive impact and the high adoption rate of social media by the millennial (Boyd, 2008). The existing review of literature indicates that higher education institutions can use social media as a potential marketing tool to attract aspirants (Gibbs, 2002; Helgesen, 2008; Hemsley-Brown and Oplatka, 2006).

Use of social media as a strategic tool for attracting aspirants to a B School is not a simple task. B schools generally struggle when it comes to coordinating and combining the traditional marketing strategies with the online strategies. This is primarily an integrated marketing communication decision. Schultz and Schultz (2004) define Integrated Marketing Communications (IMC) as strategic business process and marketing decisions that are made across multiple channels that helps drive brand communication. In research conducted by Barnes and Mattson (2009a; 2010), majority of college admissions officers agreed that social media is a strategic tool for recruitment of students.

The existing literature indicates that students who aspire to join B Schools or higher education institutions are generally interested in collaborating and connecting with their peers who are also aspiring to seek admissions in B Schools (Lindbeck and Fodrey, 2010). There is literature which also shows that students associate negatively with intrusive marketing messages ( $\mathrm{Li}$ et al., 2002; McCoy et al., 2007; Ranchhod, 2007). Peruta and Helm (2018a) in their research found that higher education institutions that maintain Facebook platform are effective in managing student recruitment. Literature also indicates that the strength of a university's Facebook community is positively correlated with people identifying with the university community. The research indicates that levels of trust and engagement in real time is also positively correlated with university's Facebook page engagement (Nevzat et al., 2016).

There are several digital content management strategies which are taken by B Schools. In some cases the Facebook pages is managed by outsourced vendors and in others it is managed in house by a group of students or staff or a combination of them. The strategy adopted to post messages on the official Facebook page of the B School also differ significantly from one B School to other. A scanning of official Facebook pages of the B Schools clearly show different patterns. Many schools post contents in a spontaneous manner. The content reflected and informed all about the B School activities, the events on campus without any forethought or strategy. There were some B Schools which had a planned content calendar and adopted a strategy to post content. This ensured strategic release of messages to audience in a strategic manner with a lot of forethought.

While social media is getting matured, many $\mathrm{B}$ Schools, like the corporate houses, still lack a strategic direction in using the social platform for communication (De Clerck, 2013). While there is existing literature which indicates on what students prefer to see on social media, they are narrow and they have not examined the use of social media in an engagement perspective and in a holistic fashion. This paper provides strategies on how a B School can better compose social media content to improve engagement, to better market their offerings to prospective students and also provides strategies to increase the reach of the posts. The paper follows the following sequence as shown in Fig. 1.

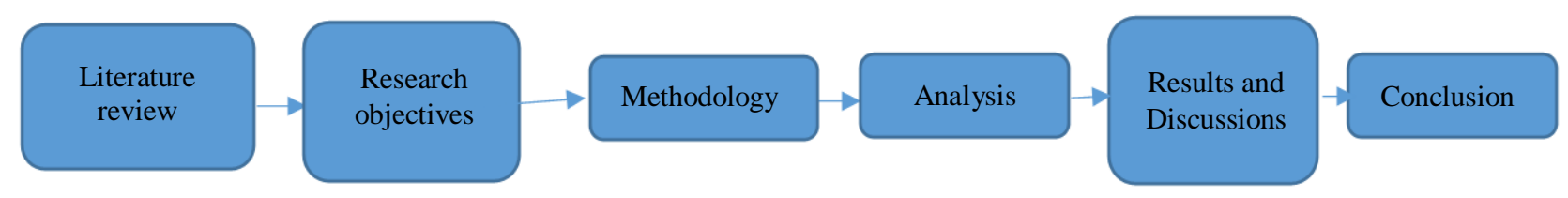

Fig. 1: The process followed 


\section{Literature Review}

\section{Indian Higher Education and Business School Landscape}

The higher education in India is a complex to understand. There are several regulatory bodies that regulate the higher education in India. Universities awarding their own degrees are classified into five types and it is based on the management that controls. The five categories are Central University, State University, Private University, Institutions-deemed-to-be-aUniversity and Institute of National Importance. In addition to this colleges award degrees in the name of the university to which they are affiliated. Apart from this there are 15 professional councils like Medical Council of India (MCI), All India Council for Technical Education (AICTE), Bar Council of India (BCI), National Skill Development Council (NSDC), Indian Nursing Council (INC) etc. that regulate the professional and skill based courses run by the colleges and universities. The University Grants Commission (UGC) acts as supreme regulatory body.

The University Grants Commission of India (UGC India) is a statutory body set up by the Indian Union government in accordance to the UGC Act 1956 under Ministry of Human Resource Development (MHRD) and is responsible for maintenance of standards in higher education. There are two accrediting institutions National Board of Accreditation (NBA) established by AICTE and National Assessment and Accreditation Council (NAAC) established by UGC which accredit institutions. There are also other private accrediting institutions like Association to Advance Collegiate Schools of Business, also known as AACSB International and the EFMD Quality Improvement System (EQUIS) run by the European Foundation for Management Development (EFMD) that rate higher educational institutions and programmes. There are two major ranking of higher education institutions in the country which are approved by MHRD. They are the National Institutional Ranking Framework (NIRF) and Atal Ranking of Institutions on Innovation Achievements (ARIIA). Apart from these two there also other private institutions like The Times Higher Education World University Rankings, Quacquarelli Symonds (QS) ranking which rank B Schools and other higher education institutions.

National Institution Ranking Framework (NIRF) is a methodology adopted by the Ministry of Human Resource Development (MHRD), Government of India, to rank institutions of higher education in India. ARIIA is an initiative of Ministry of Human Resource Development (MHRD), Govt. of India to systematically rank all major higher educational institutions and universities in India on indicators related to
"Innovation and Entrepreneurship Development" amongst students and faculty members As the structure is very complex, it becomes all the more important for B Schools to brand and communicate with the aspirants, as it is imperative for them for their growth and survival (Harsha and Shah, 2011).

The first management program in the full time mode was started in 1957 by Andhra University. Later many private business schools were established and the government also started many IIMs across India. IIMs were established in Lucknow, Indore and Kozhikode by the end of 1990s (Sinha, 2006). In the late 90's to early 2000 there was an explosive growth of Indian B Schools and almost 1940 were functional in India by 2010 (Kumar and Dash, 2011). Post economic liberalization in 1991, there was a huge demand for qualified management graduates to run and manage business enterprises and this led to the exponential growth of B Schools across India. With the growth in the middleclass and the a competitive job market made it possible for B School to be looked at as a business opportunity by affluent investors and this fuelled a bubble in the business education market space (Shah, 2012).

As of March 2019 there are 20 IIMs and more than 3500 B Schools in India. IIMs select students based on an all India entrance examinations named Common Aptitude Test (CAT) and a few hundred million appear for the examination and this happens due to the pride associated with pursuing a master in business administration from these premier institution. There are also several reputed private B Schools in the country which accept the scores of CAT but there are others who have their own examinations. Xavier Aptitude Test (XAT) and Symbiosis National Aptitude Test (SNAP). These examinations also attracts a few hundred million students who take up the exam with an aspiration to join the top private $b$ schools in the country.

Getting admitted in a top Indian B School is always a dream for many aspirants who plan to pursue their management program. The prime driving factors for this is -almost sure of securing a dream job after the program. Excluding the Indian administrative and foreign services, a management degree ranks first in the pecking order by aspirants who plan to take up professional career (Philip, 2008). Although there are many who aspire to join the top rung B Schools, many private B Schools try to attract the best student talent to join them. The Indian B School market is a highly cluttered with the presence of several $b$ schools that are private autonomous, promoted by state and central universities, independent government supported and those promoted by corporations (Sinha, 2006).

With the increase in the number of private $B$ Schools with relatively high fee that is charged by the private players, all the top private B Schools spend a 
few thousand millions in advertisement and branding, to communicate about their school to the prospective aspirants. This is done to ensure that the prospective aspirants choose their school over the others. The IMC spends are both on offline and online, with the online spends taking a higher share. As the market is competitive and with many foreign players entering the B school landscape, it is important for b schools to use innovative social media tools to engage and attract the best aspirants to apply and join the management program offered.

\section{Marketing and Social Media}

Social media remains one of the sought after online interactive platform used by B Schools to interact with aspirants who plan to join them. Like many other social media platform, Facebook also runs on the content generated by or shared by the users and hence allows peer-to-peer communication and participation (Nambisan and Nambisan, 2008). With high levels of mobile and internet penetration in India, social media has been widely used by millennial and has become a vital factor that influences their decisions and buying behavior. Literature also indicates that perceived ease of use and social impact are significant dimensions of technology acceptance and inclination to use mobile based applications (Rakhi and Mala, 2013) Although there are many social media sites, perceived usefulness of the site by the users is a significant mediating factor for the social networking site to be accepted by the user . Research indicates that online trust has a significant impact on the customer purchase intention (Arun and Xavier, 2013). As the content is generated by the users and the platform allows peer-to-peer communication, the trust in social media platforms is higher when compared to other traditional forms of marketing communication (Eikelmann et al., 2008; Gronroos, 1994; Karin and Eiferman, 2006; Peppers and Rogers, 1993; Thomas, 2007). This is a trend pattern originated in the 1990s (Gronroos, 1994; Peppers and Rogers, 1993). The behavior towards a product or service is also influenced by social media. In a research study on social media and user engagement by Apoorva et al. (2013), various aspect related to the relation between content present in the social media and the user engagement was statistically analyzed.

Literature review provides evidence that many organisations and higher educational institutions are engaging and using social media as an integral part of their marketing strategy (Barnes and Mattson, 2009a; $2009 b$ ). The content generated by users on social media is called as user generated content (Kaplan and Henlein, 2010). The social media is largely run by the content from the users, influencing the decision making process and having a full control on the purchasing behavior is sometimes beyond the control of any organisation or B School (Constantinides and Fountain, 2008).

B Schools are also aware that social media and platform like Facebook have increased the market transparency. This has made higher educational institutions to lose the control of the content that is pushed for advertisement or the content for news. The control is also lost for the communication message that is pushed to the aspirants. B Schools are hence forced to find innovative ways to reach aspirants and also find innovative ways to communicate and push content on the social platform that are used by the aspirants. Hence social media marketing for B Schools is successful only if it is focused on being innovative and has a market oriented approach and has a well-designed strategy (Constantinides, 2010).

\section{Social Media Marketing in Higher Education Institutions}

Several decades ago academia and higher educational institutions once thought 'Marketing' themselves as something that should not be publicly done or spoken about (Edmiston-Strasser, 2009). In the past, as competition was not intense, marketing of educational institutions was not a 'need' and hence did not receive support from promoters. The primary objection against marketing of higher education institutions was the aspect that it would lower the quality of academic standards (Anderson, 2008). Several researchers have concluded that due to increase in competition and changing regulations by the government (Hemsley-Brown and Oplatka, 2006; Jongbloed, 2003; Maringe, 2006), higher education institutions and business school must market themselves to survive and grow in the local and global markets. As traditional marketing models are based on consumptive models, they fail to address the needs of Business schools (Gibbs, 2002). Literature suggests that higher education marketing has to be viewed from a model of 'collaborative relationships' Gibbs (2002) or a relationship marketing approach has to adopted (Helgesen, 2008; Klassen, 2002) with ethics and ethical practices being given importance (Gibbs and Murphy, 2009). Technology is changing fast. Application of Augmented Reality (AR) technologies in social marketing along with a change-driven usage of the AR environments for social marketing is also being researched (Joshi, 2018).

Literature also shows that majority of millennial find content generated by their peers on the social media platform to have higher influence on them when compared to an advertisement or a post generated by a brand (Bazaarvoice, 2012). Also the content shared by a student or a faculty member or a staff member of a B School gets eight time's higher engagement and attention when compared to the content shared by the $\mathrm{B}$ 
School media manager. (Dunay, 2014). For any B School relationship marketing is of paramount importance. Building and maintaining relationship with aspirants, its students and alumni is a must. The quality of relationship that a business school maintains with them is positively correlated to it branding and aspirant loyalty (McAlexander and Koenig, 2001). B Schools can use their websites to provide an engaging user environment (Weiss, 2008). Blogs and social media are the ideal platforms for relational marketing activities, as they are collaborative and interactive nature. Indian business schools are increasingly using social media as part of their marketing programs and universities in the United States of America have been using social media for their marketing activities (Barnes and Mattson, 2009a).

Research indicates that there is a strong positive correlation between an aspirant logging into a social network or a social media page of a university and the chance of he / she applying to a programme offered by that university (Hayes et al., 2009). In India, there are a few pioneering efforts by $\mathrm{b}$ Schools to introduce social media as part of their student recruitment programs. All private B schools web sites display links to Twitter, Facebook pages, linked page or blogs. These b schools allow visitors to share information by bookmarking pages as favorites by 'liking it' or in case of twitter they can 're-tweet' the tweets posted on the twitter page. Although some private business schools use blogs, blogging is not part of the social media mix of majority of Indian business schools.

To summarize there is little evidence from research on the actual effects of 'social media' as recruitment tool or 'the strategic approach to use of social media' for attracting aspirants by higher education institution or $\mathrm{b}$ school.

\section{Social Media - Engagements and Reach}

Literature shows that Facebook adds 500,000 new users every day and 6 new profiles every second. Facebook generates 4 new petabytes of data per day and this indicates that the volume has exploded and is growing every day (Brandwatch, 2019).

Research indicates that young connected people are increasingly watching similar content online (Madhavan et al., 2015). Facebook alters its algorithm at regular intervals and the algorithm controls the ordering and presentation of posts, so that users see what is most relevant to them. Organic reach of a content is that content (text or video or image) which is the spread of social media content, which has not been paid for or promoted. According to Adage (2018) even for the biggest brands, organic Facebook reach fell from $4 \%$ to $2 \%$ for the year 2018. This indicates that if a B school page has 100 followers on its Facebook page, an organic post (which is an unpaid or un-promoted post) will reach only 2 out of 100 followers whom it should have reached. Hence if a brand has to reach a larger number of audience then taking the paid mode is the only option to go for and boosting a Facebook post will help reach more audience. Boosting a post is done by paying money to Facebook, so that the post can be shown to more number of followers of a Facebook page, that weren't seeing the original post. The other paid mode is to advertise on Facebook. The ads will be targeted to a specific group of people at specific region and other segmentation criteria. The next question would be, if this is the case then, why should B Schools bother about organic reach? Facebook constantly makes changes to its algorithm so that users see what is most relevant to them and hence if more and more users engage with a post and the organic reach increases exponentially. Engaging with a post can be done by clicking on the post, liking the post commenting on the post or by sharing the post. Therefore early higher engagement for a post could ensure higher organic reach for the post.

There are several ways to measure engagement on Facebook. The direct way to measure engagement is the sum total of all likes, comments and share of a post which is the score given for total engagement for the post. The alternate way to measure engagement for a post is by using proportional engagement. Proportional engagement takes into account the reach for each post and the total number of Facebook fans that a page has. The value of proportional engagement is the sum total of all likes, comments and share of a post divided by the number of Facebook fans (Peruta and Shields, 2016). Research also shows that the impact that a post has is significantly affected by the number of fans following a Facebook page.

Existing literature shows that the proportional engagement largely depends on type of post. Photos and Videos are more likely to get a higher engagement when compared to simple text posts and a director post of a video or a photo gets higher proportional engagement when compared to web link or a link to a blog that has content (Kwok and Yu, 2013). The number of posts per day on a Facebook page is inversely proportional to 'proportional engagement'. Research also indicates that Facebook pages that have one or two posts in a day get $40 \%$ more user engagement than those with higher number of posts (Buddy Media, 2011). The time of the day when a post is posted on Facebook page as an impact on engagement. Literature review has different views when it comes to answering the question on when will people access their Facebook page which can result in higher engagement? According to Lee (2012) people generally check Facebook after office hours but Zarella (2011) indicates that noon or post evening could be right time for post for higher engagement. According to Brandwatch (2019) globally users on an average spend 
20 min of their time on Facebook and posts that are put up on Thursday and Friday get the highest engagement. Increasing the engagement for a post also depends on what digital call to action is expected out of the user for the post (Vahl, 2015). Literature indicates that if specific call to action similar to - like if you agree- if brought in a post, engagement increases. Literature also indicates that instilling some psychological prodding can increase the engagement for a Facebook post (Lee, 2012).

Although social media strategies for Facebook can be devised for $b$ schools by looking at the existing literature, the strategies to be adopted by business school have to be different and cannot be based only on the general indications given by literature. The primary reason for this is due to the fact that the $b$ school has a diverse set of audience including its aspirants, its present students, its alumni and the corporate houses which recruit students from them. The content that is posted by the business school must be appealing but not disgruntling any of them. This has a relation to identity theory which theory states that individuals in the present day, participate in a many differentiated relationships and have multiple selves (William, 1950) and hence will possesses a multiplicity of identities. The theory also states that the number and nature of identities are a function of the roles filled in interaction with others. Identity theory also envisages that the probability of a given identity being played out in social interaction will be ominously impacted by the salience of the identity relative to the salience of other identities the person holds (Stryker, 1968). Identity salience is defined as the chance that a given identity will be invoked in social interaction (Stryker, 1968). Hence for an aspirant the salient identity that he or she holds will play out when he or she engages with content posted on a Facebook page by a business school. Literature also reveals that posts on Facebook by higher education institution that promote the idea of a university which is beyond its own boundaries, which has a significant impact on the society or has content that relates to the social good for the world can increase engagement (Peruta and Helm, 2018b).

\section{Research Objectives}

While there have been studies showing the relevance of social media and its influence on a brand, there is very limited research that has been done to find the use and impact of social media on promoting a B-School in the Indian context. The objective of this research paper is to explore and find how Indian higher educational institutions, with specific reference to the business schools, use Facebook to construct and deliver social media content. The prime objectives include (a) Recognize the type and content of the message that business schools are posting on the Facebook platform. (b) Recognize the structure and format of the content that is being posted by business schools on Facebook platform (c) Gauge the engagement on the type content and the type of format (d) Explore and suggest the strategies that could be adopted for maximizing engagement on the content that is posted by business school on their Facebook page.

\section{Methodology}

A non-probabilistic sampling - purposive sampling was used for selecting the B-Schools and their Facebook pages for this study. As the objective of purposive sampling is to focus on specific characteristics of a population that are of interest, the authors choose Facebook pages of top 100 Indian B Schools. The top business school were selected by considering National Institutional Ranking Framework (NIRF) developed by Ministry of Human Resource Development Government of India - India Rankings 2018 under Management category (NIRF Ranking, 2018).

Data collected was collected the Facebook pages of the top 100 business school using Facebook Graph Application Programming Interface (API). The API was used to extract data from the face Facebook pages of the business schools that were posted between May 2018 to January 2019. The total posts that were extracted for the study was 19612. As many business schools have their academic and extracurricular activities along with the promotion of their brand during this time period, the different posting strategies used could be captured. The page level and post level data was captured for the publicly posted content which was as per the ethical code of conduct accepted and signed by the users while the setup their Facebook pages. As shown in the Table 1 several attributes were collected both at the page and post level.

The data was exported to Statistical Package for the Social Sciences (SPSS) for analyses. Only publicly available and publicly displayed post were accessed and user profile data was not accessed and all aspect which could reveal the personality trait or liking as listed by the uses in their Facebook profile were also not accessed. The data was anonymous and hence all personally identifiable information was not collected and not stored. All data was in aggregate format and were not changed or manipulated for the study. For the study the total engagement and proportional engagement were measured. Although through click data on the Facebook post is defined as engagement parameter by Facebook, it was not considered by the authors due to the fact that through click data is not accessible by public API. The authors could use the Facebook Graph API that was available till the end of January 2019. This application was revoked for public access by Facebook during the mid February 2019. 
Table 1: Attribute collection

\begin{tabular}{ll}
\hline Page level & Post level \\
\hline Name of the Business School & Post Creation date \\
Number of Page likes & Post creation time \\
& Post text \\
Post description \\
Media type posted \\
Number of Characters \\
Number of comments \\
Number of Likes \\
Number of Shares \\
Number of hashtags \\
Number of tags \\
\hline
\end{tabular}

Based on the content of the posts, they were categorized in the specific category and specific format. The categorization of the post into category and format was based on the title/subject of the post. This was a manual process as there was no ready solution available for this categorization This was primarily due to the fact that the data was highly unstructured. An initial set of 3500 posts (randomly selected from the first 6000) were used to arrive at 9 categories and 5 format types. The next set of 3500 posts (randomly selected from the next 6000) were considered to add 5 more categories and 6 format types and finally after the next random selection of another 3500 posts from the remaining, made the total count of categories as 21 and total format types as 14 . The content category and formats are shown in Table 2. The 21 categories types and 14 formats helped in placing all the posts into the respective categories and format types. The labels given to category and format explains the basis on which the categorization was carried. This activity - categorization into category and formats - was the most time consuming activity. While randomly selecting the post for arriving at the category and the format, due care was taken to ensure that the same posts are not included in the selection and SPSS select case was used to randomly select samples as elaborated.

The posts were necessary placed in specific category and format. Hence one post was placed under one category only. When there were posts which had many formats then they were placed under 'Mix mode and others' format. When posts had several information and could not be placed under specific category they were placed under 'Mix composite content and others' category.

All the posts were also categorized into two categories - weekday posts and weekend posts. Those posts that were posted between Monday and Friday were categorized as week day posts and those posts which were posted during the weekend (Saturday and Sunday) were categorized as weekend post. These details are shown in Table 3. The time of the day when the posts were posted on Facebook were also captured for all the posts.
Table 2: Category and format

\begin{tabular}{ll}
\hline Category & Format \\
\hline Admissions & Blog Post link \\
Academic & Call for action \\
Alumni & Info-graphic \\
Academic Achievement - Staff & Link to URL \\
Academic Achievement - Faculty member & Message \\
Academic Achievement- Student & Opinion \\
B School Competition & Picture \\
B School Ranking & Picture and Text \\
Campus Information and updates & Podcast \\
Case Competition by B School & Question \\
Case Competition by Corporate & User generated content \\
Extra-Curricular & Video \\
Entertainment and Events & Video and Text \\
Innovation & Mix mode and others \\
Internationalization & \\
News related to Education & \\
Placement related information & \\
Promotion of Programmes & \\
Research Related Information & \\
Startups Information & \\
Mix Composite content and Others & \\
\hline
\end{tabular}

Table 3: Day and time categorization

\begin{tabular}{|c|c|c|}
\hline Nature of post & Number of posts & Percentage \\
\hline Monday to Friday & 13338 & $68.01 \%$ \\
\hline $00.00 \mathrm{am}$ to $08.00 \mathrm{am}$ & 917 & \\
\hline 08.01 am to $10.00 \mathrm{am}$ & 1324 & \\
\hline 10.01 am to $05 \# 00 \mathrm{pm}$ & 6699 & \\
\hline $05.01 \mathrm{pm}$ to $08.00 \mathrm{pm}$ & 2312 & \\
\hline $08.01 \mathrm{pm}$ to $10.00 \mathrm{pm}$ & 1321 & \\
\hline $10.00 \mathrm{pm}$ to $11.59 \mathrm{pm}$ & 765 & \\
\hline Saturday and Sunday & 6274 & $31.99 \%$ \\
\hline $00.00 \mathrm{am}$ to $08.00 \mathrm{am}$ & 342 & \\
\hline $08.01 \mathrm{am}$ to $10.00 \mathrm{am}$ & 1321 & \\
\hline 10.01 am to $05 \# 00 \mathrm{pm}$ & 2787 & \\
\hline $05.01 \mathrm{pm}$ to $08.00 \mathrm{pm}$ & 1089 & \\
\hline $08.01 \mathrm{pm}$ to $10.00 \mathrm{pm}$ & 532 & \\
\hline $10.00 \mathrm{pm}$ to $11.59 \mathrm{pm}$ & 203 & \\
\hline
\end{tabular}

Table 4 shows the results of categorization of all 19612 posts. The number of posts in a specific category and the percentage. The categories including Admissions, Academic, Alumni, B School Ranking, B School Competition, Case Competition by Corporate, Promotion of Programmes have majority of posts. Similarly majority of the posts fall under the following formats - Call for action, Message, Picture and Text, Question, User Generated Content, Video and Text.

\section{Analysis}

To understand which category increases proportional engagement, the hypothesis was created in the following fashion for all the categories. One-way ANOVA (Analysis of Variance) test was used to understand if the proportional engagement differed based on the category of the post, as the categories were 21 in number. The value of proportional engagement is the sum total of all likes, comments and share of a post divided by the overall number of page likes. 
Table 4: Results of categorization

\begin{tabular}{|c|c|c|}
\hline Type & $\mathrm{N}$ (Number of Posts) & Percentage \\
\hline \multicolumn{3}{|l|}{ Category } \\
\hline Admissions & 1121 & $5.72 \%$ \\
\hline Academic & 1031 & $5.26 \%$ \\
\hline Alumni & 2154 & $10.98 \%$ \\
\hline Academic Achievement - Staff & 189 & $0.96 \%$ \\
\hline Academic Achievement - Faculty member & 187 & $0.95 \%$ \\
\hline Academic Achievement- Student & 2309 & $11.77 \%$ \\
\hline B School Competition & 1151 & $5.87 \%$ \\
\hline B School Ranking & 1012 & $5.16 \%$ \\
\hline Campus Information and updates & 898 & $4.58 \%$ \\
\hline Case Competition by B School & 112 & $0.57 \%$ \\
\hline Case Competition by Corporate & 1135 & $5.79 \%$ \\
\hline Extra-Curricular & 589 & $3.00 \%$ \\
\hline Entertainment and Events & 1576 & $8.04 \%$ \\
\hline Innovation & 176 & $0.90 \%$ \\
\hline Internationalization & 162 & $0.83 \%$ \\
\hline News related to Education & 642 & $3.27 \%$ \\
\hline Placement related information & 812 & $4.14 \%$ \\
\hline Promotion of Programmes & 3665 & $18.69 \%$ \\
\hline Research Related Information & 221 & $1.13 \%$ \\
\hline Startups Information & 391 & $1.99 \%$ \\
\hline Mix Composite content and Others & 79 & $0.40 \%$ \\
\hline TOTAL & 19612 & $100.00 \%$ \\
\hline \multicolumn{3}{|l|}{ Format } \\
\hline Blog Post link & 227 & $1.16 \%$ \\
\hline Call for action & 2198 & $11.21 \%$ \\
\hline Info-graphic & 1187 & $6.05 \%$ \\
\hline Link to URL & 1156 & $5.89 \%$ \\
\hline Message & 2176 & $11.10 \%$ \\
\hline Opinion & 1231 & $6.28 \%$ \\
\hline Picture & 1243 & $6.34 \%$ \\
\hline Picture and Text & 2114 & $10.78 \%$ \\
\hline Podcast & 198 & $1.01 \%$ \\
\hline Question & 2123 & $10.83 \%$ \\
\hline User Generated Content & 2134 & $10.88 \%$ \\
\hline Video & 1232 & $6.28 \%$ \\
\hline Video and Text & 2321 & $11.83 \%$ \\
\hline Mix Mode and Others & 72 & $0.37 \%$ \\
\hline TOTAL & 19612 & \\
\hline
\end{tabular}

Table 5: Descriptive information of categories of post

\begin{tabular}{|c|c|c|c|c|c|c|c|c|}
\hline \multirow[b]{2}{*}{ Category type } & \multirow[b]{2}{*}{$\mathrm{N}$} & \multirow[b]{2}{*}{ Mean } & \multirow{2}{*}{$\begin{array}{l}\text { Std. } \\
\text { Deviation }\end{array}$} & \multirow[b]{2}{*}{ Std. Error } & \multicolumn{2}{|c|}{$95 \%$ confidence interval for mean } & \multirow[b]{2}{*}{ Min } & \multirow[b]{2}{*}{$\operatorname{Max}$} \\
\hline & & & & & Lower bound & Upper bound & & \\
\hline Admissions & 100 & 0.14889 & 0.019509 & 0.001951 & 0.14502 & 0.15276 & 0.094 & 0.157 \\
\hline Alumni & 100 & 0.18739 & 0.024427 & 0.002443 & 0.18254 & 0.19223 & 0.118 & 0.197 \\
\hline Academic Achievement-Staff & 100 & 0.05937 & 0.007941 & 0.000794 & 0.05779 & 0.06095 & 0.037 & 0.064 \\
\hline $\begin{array}{l}\text { Academic Achievement - } \\
\text { Faculty member }\end{array}$ & 100 & 0.02316 & 0.003251 & 0.000325 & 0.02252 & 0.02381 & 0.014 & 0.026 \\
\hline B School Competition & 100 & 0.05838 & 0.007825 & 0.000782 & 0.05683 & 0.05994 & 0.036 & 0.062 \\
\hline B School Ranking & 100 & 0.10138 & 0.013205 & 0.001320 & 0.09876 & 0.10400 & 0.064 & 0.108 \\
\hline Campus Information and updates & 100 & 0.02486 & 0.003222 & 0.000322 & 0.02422 & 0.02550 & 0.016 & 0.028 \\
\hline Case Competition by B School & 100 & 0.19971 & 0.026438 & 0.002644 & 0.19446 & 0.20495 & 0.125 & 0.210 \\
\hline Case Competition by Corporate & 100 & 0.21758 & 0.028800 & 0.002880 & 0.21187 & 0.22330 & 0.136 & 0.229 \\
\hline Extra-Curricular & 100 & 0.14786 & 0.019431 & 0.001943 & 0.14400 & 0.15171 & 0.093 & 0.156 \\
\hline Entertainment and Events & 100 & 0.15547 & 0.020568 & 0.002057 & 0.15139 & 0.15955 & 0.097 & 0.164 \\
\hline Innovation & 100 & 0.04747 & 0.006386 & 0.000639 & 0.04620 & 0.04874 & 0.030 & 0.051 \\
\hline Internationalization & 100 & 0.06658 & 0.008788 & 0.000879 & 0.06484 & 0.06833 & 0.042 & 0.071 \\
\hline News related to Education & 100 & 0.02012 & 0.002966 & 0.000297 & 0.01953 & 0.02070 & 0.012 & 0.023 \\
\hline Placement related information & 100 & 0.20663 & 0.027749 & 0.002775 & 0.20112 & 0.21214 & 0.129 & 0.224 \\
\hline Promotion of Programmes & 100 & 0.06990 & 0.009392 & 0.000939 & 0.06803 & 0.07176 & 0.043 & 0.075 \\
\hline Research Related Information & 100 & 0.03187 & 0.004051 & 0.000405 & 0.03106 & 0.03267 & 0.021 & 0.035 \\
\hline Startups Information & 100 & 0.17107 & 0.022431 & 0.002243 & 0.16662 & 0.17552 & 0.108 & 0.180 \\
\hline Mix Composite content and Others & 100 & 0.00573 & 0.001034 & 0.000103 & 0.00552 & 0.00593 & 0.004 & 0.008 \\
\hline Total & 2100 & 0.09888 & 0.070029 & 0.001528 & 0.09588 & 0.10188 & 0.004 & 0.229 \\
\hline
\end{tabular}


The Hypothesis (A) $H_{0}$ - There is no significant difference in proportional engagement for category of the post was crafted to check if there was any significant difference in the engagement and its relation the category of the post. The descriptive statistical information given in Table 5 gives descriptive statistical information, including mean, standard deviation and $95 \%$ confidence intervals for the dependent variable- proportional engagement score- for each of the category of the Facebook post where all the 21 categories have been listed.

The Table 6 is the output of the ANOVA analysis which is used to test the Hypothesis (A) to understand if there is a statistically significant difference between the group means of proportional engagement scores and the category of the post. It can be inferred from the significance value 0.000 (i.e., $\mathrm{p}=0.000$ ), which is below 0.05 , that there is a statistically significant difference between in proportional engagement and category of the post. Which clearly indicates that different posts under different categories have different engagements. To know which of those specific categories differed in the engagement, Tukey post hoc test was used and the Table 7 gives the output which is the multiple comparisons table. From the multiple comparisons Table 7 it can be statistically inferred that there is a statistically significant difference in proportional engagement in posts that were posted in admissions category in comparison with all other categories $(\mathrm{p}=0.000)$, but there is a statistically no significant difference between the posts under the admissions category and the posts under extraCurricular $(\mathrm{p}=0.287)$ and entertainment and events categories $(\mathrm{p}=0.287)$. For the posts related to Alumni, B School Ranking, Case Competition by Corporate, startups Information and, Academic AchievementStudent content categories it can be statistically inferred that there is a statistically significant difference in proportional engagement in posts that were posted in these category in comparison with all other categories $(\mathrm{p}=0.000)$. The Table 8 gives the details for all the categories for which there is a statistically no significant difference in proportional engagement in posts when compared to other categories.

Table 6: ANOVA analysis - category type

ANOVA

Category type engagement

\begin{tabular}{lcrll}
\hline & Sum of Squares & df & Mean square & F \\
\hline Between Groups & 9.77 & 20 & 0.488 & 1940.02 \\
Within Groups & 0.523 & 2079 & 0.000 & \\
Total & 10.293 & 2099 & & \\
\hline
\end{tabular}

Table 7: Multiple comparisons of categories

\begin{tabular}{|c|c|c|c|c|c|c|}
\hline \multicolumn{7}{|c|}{$\begin{array}{l}\text { Dependent Variable: Engagement } \\
\text { Tukey HSD }\end{array}$} \\
\hline \multirow[b]{2}{*}{ (I) Category } & \multirow[b]{2}{*}{ (J) Category } & \multirow{2}{*}{$\begin{array}{l}\text { Mean } \\
\text { Difference (I-J) }\end{array}$} & \multirow[b]{2}{*}{ Std. Error } & \multirow[b]{2}{*}{ Sig. } & \multicolumn{2}{|c|}{$95 \%$ Confidence Interval } \\
\hline & & & & & Lower bound & Upper bound \\
\hline \multirow[t]{20}{*}{ Admissions } & Academic & $0.094092^{*}$ & 0.002244 & 0.000 & 0.08607 & 0.10211 \\
\hline & Alumni & $-0.038499^{*}$ & 0.002244 & 0.000 & -0.04652 & -0.03048 \\
\hline & Academic Achievement - Staff & $0.089517^{*}$ & 0.002244 & 0.000 & 0.08150 & 0.09754 \\
\hline & Academic Achievement - Faculty member & $0.125724^{*}$ & 0.002244 & 0.000 & 0.11770 & 0.13374 \\
\hline & Academic Achievement- Student & $0.070634^{*}$ & 0.002244 & 0.000 & 0.06261 & 0.07865 \\
\hline & B School Competition & $0.090503^{*}$ & 0.002244 & 0.000 & 0.08248 & 0.09852 \\
\hline & B School Ranking & $0.047505^{*}$ & 0.002244 & 0.000 & 0.03949 & 0.05552 \\
\hline & Campus Information and updates & $0.124028^{*}$ & 0.002244 & 0.000 & 0.11601 & 0.13205 \\
\hline & Case Competition by B School & $-0.050822^{*}$ & 0.002244 & 0.000 & -0.05884 & -0.04280 \\
\hline & Case Competition by Corporate & $-0.068695^{*}$ & 0.002244 & 0.000 & -0.07671 & -0.06068 \\
\hline & Extra-Curricular & 0.001030 & 0.002244 & 0.287 & -0.00699 & 0.00905 \\
\hline & Entertainment and Events & -0.006586 & 0.002244 & 0.287 & -0.01461 & 0.00143 \\
\hline & Innovation & $0.101419^{*}$ & 0.002244 & 0.000 & 0.09340 & 0.10944 \\
\hline & Internationalization & $0.082303^{*}$ & 0.002244 & 0.000 & 0.07428 & 0.09032 \\
\hline & News related to Education & $0.128771^{*}$ & 0.002244 & 0.000 & 0.12075 & 0.13679 \\
\hline & Placement related information & $-0.057744^{*}$ & 0.002244 & 0.000 & -0.06576 & -0.04972 \\
\hline & Promotion of Programmes & $0.078989^{*}$ & 0.002244 & 0.000 & 0.07097 & 0.08701 \\
\hline & Research Related Information & $0.117019^{*}$ & 0.002244 & 0.000 & 0.10900 & 0.12504 \\
\hline & Startups Information & $-0.022179^{*}$ & 0.002244 & 0.000 & -0.03020 & -0.01416 \\
\hline & Mix Composite content and Others & $0.143161^{*}$ & 0.002244 & 0.000 & 0.13514 & 0.15118 \\
\hline \multirow[t]{4}{*}{ Academic } & Admissions & $-0.094092^{*}$ & 0.002244 & 0.000 & -0.10211 & -0.08607 \\
\hline & Alumni & $-0.132591^{*}$ & 0.002244 & 0.000 & -0.14061 & -0.12457 \\
\hline & Academic Achievement - Staff & -0.004575 & 0.002244 & 0.901 & -0.01259 & 0.00344 \\
\hline & Academic Achievement - Faculty member & $0.031632^{*}$ & 0.002244 & 0.000 & 0.02361 & 0.03965 \\
\hline
\end{tabular}


Vikram Kumar and Ramakrishnan Raman / Journal of Computer Science 2019, 15 (9): 1256.1282 DOI: 10.3844/jessp.2019.1256.1282

\begin{tabular}{|c|c|c|c|c|c|c|}
\hline & Academic Achievement- Student & $-0.023458^{*}$ & 0.002244 & 0.000 & -0.03148 & -0.01544 \\
\hline & B School Competition & -0.003589 & 0.002244 & 0.992 & -0.01161 & 0.00443 \\
\hline & B School Ranking & $-0.046587^{*}$ & 0.002244 & 0.000 & -0.05461 & -0.03857 \\
\hline & Campus Information and updates & $0.029936^{*}$ & 0.002244 & 0.000 & 0.02192 & 0.03796 \\
\hline & Case Competition by B School & $-0.144914^{*}$ & 0.002244 & 0.000 & -0.15293 & -0.13689 \\
\hline & Case Competition by Corporate & $-0.162787^{*}$ & 0.002244 & 0.000 & -0.17081 & -0.15477 \\
\hline & Extra-Curricular & $-0.093062^{*}$ & 0.002244 & 0.000 & -0.10108 & -0.08504 \\
\hline & Entertainment and Events & $-0.100678^{*}$ & 0.002244 & 0.000 & -0.10870 & -0.09266 \\
\hline & Innovation & 0.007327 & 0.002244 & 0.127 & -0.00069 & 0.01535 \\
\hline & Internationalization & $-0.011789^{*}$ & 0.002244 & 0.000 & -0.01981 & -0.00377 \\
\hline & News related to Education & $0.034679^{*}$ & 0.002244 & 0.000 & 0.02666 & 0.04270 \\
\hline & Placement related information & $-0.151836^{*}$ & 0.002244 & 0.000 & -0.15986 & -0.14382 \\
\hline & Promotion of Programmes & $-0.015103^{*}$ & 0.002244 & 0.000 & -0.02312 & -0.00708 \\
\hline & Research Related Information & $0.022927^{*}$ & 0.002244 & 0.000 & 0.01491 & 0.03095 \\
\hline & Startups Information & $-0.116271^{*}$ & 0.002244 & 0.000 & -0.12429 & -0.10825 \\
\hline & Mix Composite content and Others & $0.049069^{*}$ & 0.002244 & 0.000 & 0.04105 & 0.05709 \\
\hline & Academic Achievement - Staff & $0.128016^{*}$ & 0.002244 & 0.000 & 0.12000 & 0.13604 \\
\hline & Academic Achievement - Faculty member & $0.164223^{*}$ & 0.002244 & 0.000 & 0.15620 & 0.17224 \\
\hline & Academic Achievement-Student & $0.109133^{*}$ & 0.002244 & 0.000 & 0.10111 & 0.11715 \\
\hline & B School Competition & $0.129002^{*}$ & 0.002244 & 0.000 & 0.12098 & 0.13702 \\
\hline & B School Ranking & $0.086004^{*}$ & 0.002244 & 0.000 & 0.07798 & 0.09402 \\
\hline & Campus Information and updates & $0.162527^{*}$ & 0.002244 & 0.000 & 0.15451 & 0.17055 \\
\hline & Case Competition by B School & $-0.012323^{*}$ & 0.002244 & 0.000 & -0.02034 & -0.00430 \\
\hline & Case Competition by Corporate & $-0.030196^{*}$ & 0.002244 & 0.000 & -0.03822 & -0.02218 \\
\hline & Extra-Curricular & $0.039529^{*}$ & 0.002244 & 0.000 & 0.03151 & 0.04755 \\
\hline & Entertainment and Events & $0.031913^{*}$ & 0.002244 & 0.000 & 0.02389 & 0.03993 \\
\hline & Innovation & $0.139918^{*}$ & 0.002244 & 0.000 & 0.13190 & 0.14794 \\
\hline & Internationalization & $0.120802^{*}$ & 0.002244 & 0.000 & 0.11278 & 0.12882 \\
\hline & News related to Education & $0.167270^{*}$ & 0.002244 & 0.000 & 0.15925 & 0.17529 \\
\hline & Placement related information & $-0.019245^{*}$ & 0.002244 & 0.000 & -0.02726 & -0.01123 \\
\hline & Promotion of Programmes & $0.117488^{*}$ & 0.002244 & 0.000 & 0.10947 & 0.12551 \\
\hline & Research Related Information & $0.155518^{*}$ & 0.002244 & 0.000 & 0.14750 & 0.16354 \\
\hline \multirow{20}{*}{$\begin{array}{l}\text { Academic } \\
\text { achievement - staff }\end{array}$} & Admissions & $-0.089517^{*}$ & 0.002244 & 0.000 & -0.09754 & -0.08150 \\
\hline & Academic & 0.004575 & 0.002244 & 0.901 & -0.00344 & 0.01259 \\
\hline & Alumni & $-0.128016^{*}$ & 0.002244 & 0.000 & -0.13604 & -0.12000 \\
\hline & Academic Achievement - Faculty member & $0.036207^{*}$ & 0.002244 & 0.000 & 0.02819 & 0.04423 \\
\hline & Academic Achievement- Student & $-0.018883^{*}$ & 0.002244 & 0.000 & -0.02690 & -0.01086 \\
\hline & B School Competition & 0.000986 & 0.002244 & 0.992 & -0.00703 & 0.00901 \\
\hline & B School Ranking & $-0.042012^{*}$ & 0.002244 & 0.000 & -0.05003 & -0.03399 \\
\hline & Campus Information and updates & $0.034511^{*}$ & 0.002244 & 0.000 & 0.02649 & 0.04253 \\
\hline & Case Competition by B School & $-0.140339^{*}$ & 0.002244 & 0.000 & -0.14836 & -0.13232 \\
\hline & Case Competition by Corporate & $-0.158212^{*}$ & 0.002244 & 0.000 & -0.16623 & -0.15019 \\
\hline & Extra-Curricular & $-0.088487^{*}$ & 0.002244 & 0.000 & -0.09651 & -0.08047 \\
\hline & Entertainment and Events & $-0.096103^{*}$ & 0.002244 & 0.000 & -0.10412 & -0.08808 \\
\hline & Innovation & $0.011902^{*}$ & 0.002244 & 0.000 & 0.00388 & 0.01992 \\
\hline & Internationalization & -0.007214 & 0.002244 & 0.146 & -0.01523 & 0.00081 \\
\hline & News related to Education & $0.039254^{*}$ & 0.002244 & 0.000 & 0.03123 & 0.04727 \\
\hline & Placement related information & $-0.147261^{*}$ & 0.002244 & 0.000 & -0.15528 & -0.13924 \\
\hline & Promotion of Programmes & $-0.010528^{*}$ & 0.002244 & 0.001 & -0.01855 & -0.00251 \\
\hline & Research Related Information & $0.027502^{*}$ & 0.002244 & 0.000 & 0.01948 & 0.03552 \\
\hline & Startups Information & $-0.111696^{*}$ & 0.002244 & 0.000 & -0.11972 & -0.10368 \\
\hline & Mix Composite content and Others & $0.053644^{*}$ & 0.002244 & 0.000 & 0.04562 & 0.06166 \\
\hline \multirow{12}{*}{$\begin{array}{l}\text { Academic } \\
\text { achievement - faculty } \\
\text { member }\end{array}$} & Admissions & $-0.125724^{*}$ & 0.002244 & 0.000 & -0.13374 & -0.11770 \\
\hline & Academic & $-0.031632^{*}$ & 0.002244 & 0.000 & -0.03965 & -0.02361 \\
\hline & Alumni & $-0.164223^{*}$ & 0.002244 & 0.000 & -0.17224 & -0.15620 \\
\hline & Academic Achievement - Staff & $-0.036207^{*}$ & 0.002244 & 0.000 & -0.04423 & -0.02819 \\
\hline & Academic Achievement- Student & $-0.055090^{*}$ & 0.002244 & 0.000 & -0.06311 & -0.04707 \\
\hline & B School Competition & $-0.035221^{*}$ & 0.002244 & 0.000 & -0.04324 & -0.02720 \\
\hline & B School Ranking & $-0.078219^{*}$ & 0.002244 & 0.000 & -0.08624 & -0.07020 \\
\hline & Campus Information and updates & -0.001696 & 0.002244 & 0.877 & -0.00972 & 0.00632 \\
\hline & Case Competition by B School & $-0.176546^{*}$ & 0.002244 & 0.000 & -0.18457 & -0.16853 \\
\hline & Case Competition by Corporate & $-0.194419^{*}$ & 0.002244 & 0.000 & -0.20244 & -0.18640 \\
\hline & Extra-Curricular & $-0.124694^{*}$ & 0.002244 & 0.000 & -0.13271 & -0.11667 \\
\hline & Entertainment and Events & $-0.132310^{*}$ & 0.002244 & 0.000 & -0.14033 & -0.12429 \\
\hline
\end{tabular}


Vikram Kumar and Ramakrishnan Raman / Journal of Computer Science 2019, 15 (9): 1256.1282 DOI: 10.3844/jessp.2019.1256.1282

\begin{tabular}{|c|c|c|c|c|c|c|}
\hline \multirow{26}{*}{$\begin{array}{l}\text { Academic } \\
\text { achievement-student }\end{array}$} & Innovation & $-0.024305^{*}$ & 0.002244 & 0.000 & -0.03232 & -0.01629 \\
\hline & Internationalization & $-0.043421^{*}$ & 0.002244 & 0.000 & -0.05144 & -0.03540 \\
\hline & News related to Education & 0.003047 & 0.002244 & 0.992 & -0.00497 & 0.01107 \\
\hline & Placement related information & $-0.183468^{*}$ & 0.002244 & 0.000 & -0.19149 & -0.17545 \\
\hline & Promotion of Programmes & $-0.046735^{*}$ & 0.002244 & 0.000 & -0.05475 & -0.03872 \\
\hline & Research Related Information & $-0.008705^{*}$ & 0.002244 & 0.017 & -0.01672 & -0.00069 \\
\hline & Startups Information & $-0.147903^{*}$ & 0.002244 & 0.000 & -0.15592 & -0.13988 \\
\hline & Admissions & $-0.070634^{*}$ & 0.002244 & 0.000 & -0.07865 & -0.06261 \\
\hline & Academic & $0.023458^{*}$ & 0.002244 & 0.000 & 0.01544 & 0.03148 \\
\hline & Alumni & $-0.109133^{*}$ & 0.002244 & 0.000 & -0.11715 & -0.10111 \\
\hline & Academic Achievement - Staff & $0.018883^{*}$ & 0.002244 & 0.000 & 0.01086 & 0.02690 \\
\hline & Academic Achievement - Faculty member & $0.055090^{*}$ & 0.002244 & 0.000 & 0.04707 & 0.06311 \\
\hline & B School Competition & $0.019869^{*}$ & 0.002244 & 0.000 & 0.01185 & 0.02789 \\
\hline & B School Ranking & $-0.023129^{*}$ & 0.002244 & 0.000 & -0.03115 & -0.01511 \\
\hline & Case Competition by B School & $-0.121456^{*}$ & 0.002244 & 0.000 & -0.12948 & -0.11344 \\
\hline & Case Competition by Corporate & $-0.139329^{*}$ & 0.002244 & 0.000 & -0.14735 & -0.13131 \\
\hline & Extra-Curricular & $-0.069604^{*}$ & 0.002244 & 0.000 & -0.07762 & -0.06158 \\
\hline & Entertainment and Events & $-0.077220^{*}$ & 0.002244 & 0.000 & -0.08524 & -0.06920 \\
\hline & Innovation & $0.030785^{*}$ & 0.002244 & 0.000 & 0.02277 & 0.03880 \\
\hline & Internationalization & $0.011669^{*}$ & 0.002244 & 0.000 & 0.00365 & 0.01969 \\
\hline & News related to Education & $0.058137^{*}$ & 0.002244 & 0.000 & 0.05012 & 0.06616 \\
\hline & Placement related information & $-0.128378^{*}$ & 0.002244 & 0.000 & -0.13640 & -0.12036 \\
\hline & Promotion of Programmes & $0.008355^{*}$ & 0.002244 & 0.030 & 0.00034 & 0.01637 \\
\hline & Research Related Information & $0.046385^{*}$ & 0.002244 & 0.000 & 0.03837 & 0.05440 \\
\hline & Startups Information & $-0.092813^{*}$ & 0.002244 & 0.000 & -0.10083 & -0.08479 \\
\hline & Mix Composite content and Others & $0.072527^{*}$ & 0.002244 & 0.000 & 0.06451 & 0.08055 \\
\hline \multirow[t]{18}{*}{ B School competition } & Admissions & $-0.090503^{*}$ & 0.002244 & 0.000 & -0.09852 & -0.08248 \\
\hline & Academic & 0.003589 & 0.002244 & 0.992 & -0.00443 & 0.01161 \\
\hline & Alumni & $-0.129002^{*}$ & 0.002244 & 0.000 & -0.13702 & -0.12098 \\
\hline & Academic Achievement- Student & $-0.019869^{*}$ & 0.002244 & 0.000 & -0.02789 & -0.01185 \\
\hline & B School Ranking & $-0.042998^{*}$ & 0.002244 & 0.000 & -0.05102 & -0.03498 \\
\hline & Campus Information and updates & $0.033525^{*}$ & 0.002244 & 0.000 & 0.02551 & 0.04154 \\
\hline & Case Competition by B School & $-0.141325^{*}$ & 0.002244 & 0.000 & -0.14934 & -0.13331 \\
\hline & Case Competition by Corporate & $-0.159198^{*}$ & 0.002244 & 0.000 & -0.16722 & -0.15118 \\
\hline & Extra-Curricular & $-0.089473^{*}$ & 0.002244 & 0.000 & -0.09749 & -0.08145 \\
\hline & Entertainment and Events & $-0.097089^{*}$ & 0.002244 & 0.000 & -0.10511 & -0.08907 \\
\hline & Innovation & $0.010916^{*}$ & 0.002244 & 0.000 & 0.00290 & 0.01894 \\
\hline & Internationalization & $-0.008200^{*}$ & 0.002244 & 0.038 & -0.01622 & -0.00018 \\
\hline & News related to Education & $0.038268^{*}$ & 0.002244 & 0.000 & 0.03025 & 0.04629 \\
\hline & Placement related information & $-0.148247^{*}$ & 0.002244 & 0.000 & -0.15627 & -0.14023 \\
\hline & Promotion of Programmes & $-0.011514^{*}$ & 0.002244 & 0.000 & -0.01953 & -0.00349 \\
\hline & Research Related Information & $0.026516^{*}$ & 0.002244 & 0.000 & 0.01850 & 0.03454 \\
\hline & Startups Information & $-0.112682^{*}$ & 0.002244 & 0.000 & -0.12070 & -0.10466 \\
\hline & Mix Composite content and Others & $0.052658^{*}$ & 0.002244 & 0.060 & 0.04464 & 0.06068 \\
\hline \multirow[t]{15}{*}{ B School ranking } & Admissions & $-0.047505^{*}$ & 0.002244 & 0.000 & -0.05552 & -0.03949 \\
\hline & Academic & $0.046587^{*}$ & 0.002244 & 0.000 & 0.03857 & 0.05461 \\
\hline & Alumni & $-0.086004^{*}$ & 0.002244 & 0.000 & -0.09402 & -0.07798 \\
\hline & Academic Achievement - Staff & $0.042012^{*}$ & 0.002244 & 0.000 & 0.03399 & 0.05003 \\
\hline & Academic Achievement - Faculty member & $0.078219^{*}$ & 0.002244 & 0.000 & 0.07020 & 0.08624 \\
\hline & Academic Achievement-Student & $0.023129^{*}$ & 0.002244 & 0.000 & 0.01511 & 0.03115 \\
\hline & B School Competition & $0.042998^{*}$ & 0.002244 & 0.000 & 0.03498 & 0.05102 \\
\hline & Campus Information and updates & $0.076523^{*}$ & 0.002244 & 0.000 & 0.06850 & 0.08454 \\
\hline & Case Competition by B School & $-0.098327^{*}$ & 0.002244 & 0.000 & -0.10635 & -0.09031 \\
\hline & Case Competition by Corporate & $-0.116200^{*}$ & 0.002244 & 0.000 & -0.12422 & -0.10818 \\
\hline & Extra-Curricular & $-0.046475^{*}$ & 0.002244 & 0.000 & -0.05449 & -0.03846 \\
\hline & Entertainment and Events & $-0.054091^{*}$ & 0.002244 & 0.000 & -0.06211 & -0.04607 \\
\hline & Innovation & $0.053914^{*}$ & 0.002244 & 0.000 & 0.04589 & 0.06193 \\
\hline & Internationalization & $0.034798^{*}$ & 0.002244 & 0.000 & 0.02678 & 0.04282 \\
\hline & News related to Education & $0.081266^{*}$ & 0.002244 & 0.000 & 0.07325 & 0.08929 \\
\hline
\end{tabular}


Vikram Kumar and Ramakrishnan Raman / Journal of Computer Science 2019, 15 (9): 1256.1282 DOI: 10.3844/jessp.2019.1256.1282

Table 7: Continue

Case competition by B School
Case competition by corporate
Campus Information and updates

\begin{tabular}{|c|c|c|c|c|c|c|}
\hline \multirow{24}{*}{$\begin{array}{l}\text { Campus Information } \\
\text { and updates }\end{array}$} & Placement related information & $-0.105249^{*}$ & 0.002244 & 0.000 & -0.11327 & -0.09723 \\
\hline & Promotion of Programmes & $0.031484^{*}$ & 0.002244 & 0.000 & 0.02346 & 0.03950 \\
\hline & Research Related Information & $0.069514^{*}$ & 0.002244 & 0.000 & 0.06149 & 0.07753 \\
\hline & Startups Information & $-0.069684^{*}$ & 0.002244 & 0.000 & -0.07770 & -0.06166 \\
\hline & Mix Composite content and Others & $0.095656^{*}$ & 0.002244 & 0.000 & 0.08764 & 0.10368 \\
\hline & Admissions & $-0.124028^{*}$ & 0.002244 & 0.000 & -0.13205 & -0.11601 \\
\hline & Academic & $-0.029936^{*}$ & 0.002244 & 0.000 & -0.03796 & -0.02192 \\
\hline & Alumni & $-0.162527^{*}$ & 0.002244 & 0.000 & -0.17055 & -0.15451 \\
\hline & Academic Achievement - Staff & $-0.034511^{*}$ & 0.002244 & 0.000 & -0.04253 & -0.02649 \\
\hline & Academic Achievement - Faculty member & 0.001696 & 0.002244 & 0.877 & -0.00632 & 0.00972 \\
\hline & Academic Achievement- Student & $-0.053394^{*}$ & 0.002244 & 0.000 & -0.06141 & -0.04537 \\
\hline & B School Competition & $-0.033525^{*}$ & 0.002244 & 0.000 & -0.04154 & -0.02551 \\
\hline & B School Ranking & $-0.076523^{*}$ & 0.002244 & 0.000 & -0.08454 & -0.06850 \\
\hline & Case Competition by B School & $-0.174850^{*}$ & 0.002244 & 0.000 & -0.18287 & -0.16683 \\
\hline & Case Competition by Corporate & $-0.192723^{*}$ & 0.002244 & 0.000 & -0.20074 & -0.18470 \\
\hline & Extra-Curricular & $-0.122998^{*}$ & 0.002244 & 0.000 & -0.13102 & -0.11498 \\
\hline & Innovation & $-0.022609^{*}$ & 0.002244 & 0.000 & -0.03063 & -0.01459 \\
\hline & Internationalization & $-0.041725^{*}$ & 0.002244 & 0.000 & -0.04974 & -0.03371 \\
\hline & News related to Education & 0.004743 & 0.002244 & 0.867 & -0.00328 & 0.01276 \\
\hline & Placement related information & $-0.181772^{*}$ & 0.002244 & 0.203 & -0.18979 & -0.17375 \\
\hline & Promotion of Programmes & $-0.045039^{*}$ & 0.002244 & 0.000 & -0.05306 & -0.03702 \\
\hline & Research Related Information & -0.007009 & 0.002244 & 0.185 & -0.01503 & 0.00101 \\
\hline & Startups Information & $-0.146207^{*}$ & 0.002244 & 0.000 & -0.15423 & -0.13819 \\
\hline & Mix Composite content and Others & $0.019133^{*}$ & 0.002244 & 0.000 & 0.01111 & 0.02715 \\
\hline \multirow{18}{*}{$\begin{array}{l}\text { Case competition } \\
\text { by B School }\end{array}$} & Admissions & $0.050822^{*}$ & 0.002244 & 0.000 & 0.04280 & 0.05884 \\
\hline & Academic & $0.144914^{*}$ & 0.002244 & 0.000 & 0.13689 & 0.15293 \\
\hline & Alumni & $0.012323^{*}$ & 0.002244 & 0.000 & 0.00430 & 0.02034 \\
\hline & Academic Achievement - Staff & $0.140339^{*}$ & 0.002244 & 0.000 & 0.13232 & 0.14836 \\
\hline & Academic Achievement - Faculty member & $0.176546^{*}$ & 0.002244 & 0.000 & 0.16853 & 0.18457 \\
\hline & Academic Achievement- Student & $0.121456^{*}$ & 0.002244 & 0.000 & 0.11344 & 0.12948 \\
\hline & B School Competition & $0.141325^{*}$ & 0.002244 & 0.000 & 0.13331 & 0.14934 \\
\hline & Case Competition by Corporate & $-0.017873^{*}$ & 0.002244 & 0.000 & -0.02589 & -0.00985 \\
\hline & Extra-Curricular & $0.051852^{*}$ & 0.002244 & 0.000 & 0.04383 & 0.05987 \\
\hline & Entertainment and Events & $0.044236^{*}$ & 0.002244 & 0.000 & 0.03622 & 0.05226 \\
\hline & Innovation & $0.152241^{*}$ & 0.002244 & 0.000 & 0.14422 & 0.16026 \\
\hline & Internationalization & $0.133125^{*}$ & 0.002244 & 0.000 & 0.12511 & 0.14114 \\
\hline & News related to Education & $0.179593^{*}$ & 0.002244 & 0.000 & 0.17157 & 0.18761 \\
\hline & Placement related information & -0.006922 & 0.002244 & 0.203 & -0.01494 & 0.00110 \\
\hline & Promotion of Programmes & $0.129811^{*}$ & 0.002244 & 0.000 & 0.12179 & 0.13783 \\
\hline & Research Related Information & $0.167841^{*}$ & 0.002244 & 0.000 & 0.15982 & 0.17586 \\
\hline & Startups Information & $0.028643^{*}$ & 0.002244 & 0.000 & 0.02062 & 0.03666 \\
\hline & Mix Composite content and Others & $0.193983^{*}$ & 0.002244 & 0.000 & 0.18596 & 0.20200 \\
\hline \multirow{19}{*}{$\begin{array}{l}\text { Case competition } \\
\text { by corporate }\end{array}$} & Admissions & $0.068695^{*}$ & 0.002244 & 0.000 & 0.06068 & 0.07671 \\
\hline & Academic & $0.162787^{*}$ & 0.002244 & 0.000 & 0.15477 & 0.17081 \\
\hline & Alumni & $0.030196^{*}$ & 0.002244 & 0.000 & 0.02218 & 0.03822 \\
\hline & Academic Achievement - Staff & $0.158212^{*}$ & 0.002244 & 0.000 & 0.15019 & 0.16623 \\
\hline & Academic Achievement - Faculty member & $0.194419^{*}$ & 0.002244 & 0.000 & 0.18640 & 0.20244 \\
\hline & Academic Achievement- Student & $0.139329^{*}$ & 0.002244 & 0.000 & 0.13131 & 0.14735 \\
\hline & B School Competition & $0.159198^{*}$ & 0.002244 & 0.000 & 0.15118 & 0.16722 \\
\hline & B School Ranking & $0.116200^{*}$ & 0.002244 & 0.000 & 0.10818 & 0.12422 \\
\hline & Campus Information and updates & $0.192723^{*}$ & 0.002244 & 0.000 & 0.18470 & 0.20074 \\
\hline & Case Competition by B School & $0.017873^{*}$ & 0.002244 & 0.000 & 0.00985 & 0.02589 \\
\hline & Extra-Curricular & $0.069725^{*}$ & 0.002244 & 0.000 & 0.06171 & 0.07774 \\
\hline & Entertainment and Events & $0.062109^{*}$ & 0.002244 & 0.000 & 0.05409 & 0.07013 \\
\hline & Innovation & $0.170114^{*}$ & 0.002244 & 0.000 & 0.16209 & 0.17813 \\
\hline & Internationalization & $0.150998^{*}$ & 0.002244 & 0.000 & 0.14298 & 0.15902 \\
\hline & News related to Education & $0.197466^{*}$ & 0.002244 & 0.000 & 0.18945 & 0.20549 \\
\hline & Placement related information & $0.010951^{*}$ & 0.002244 & 0.000 & 0.00293 & 0.01897 \\
\hline & Promotion of Programmes & $0.147684^{*}$ & 0.002244 & 0.000 & 0.13966 & 0.15570 \\
\hline & Research Related Information & $0.185714^{*}$ & 0.002244 & 0.000 & 0.17769 & 0.19373 \\
\hline & Startups Information & $0.046516^{*}$ & 0.002244 & 0.000 & 0.03850 & 0.05454 \\
\hline
\end{tabular}


Vikram Kumar and Ramakrishnan Raman / Journal of Computer Science 2019, 15 (9): 1256.1282 DOI: 10.3844/jessp.2019.1256.1282

Table 7: Continue

Entertainment and events

Innovation

Internationalization
Mix Composite content and Others

Admissions

Academic

Alumni

Academic Achievement - Staff

Academic Achievement - Faculty member

Academic Achievement- Student

B School Competition

B School Ranking

Campus Information and updates

Case Competition by B School

Case Competition by Corporate

Entertainment and Events

Innovation

Internationalization

News related to Education

Placement related information

Promotion of Programmes

Research Related Information

Startups Information

Mix Composite content and Others

Admissions

and Events Academic

Alumni

Academic Achievement - Staff

Academic Achievement - Faculty member

Academic Achievement- Student

B School Competition

B School Ranking

Campus Information and updates

Case Competition by B School

Case Competition by Corporate

Extra-Curricular

Innovation

Internationalization

News related to Education

Placement related information

Promotion of Programmes

Research Related Information

Startups Information

Mix Composite content and Others

Admissions

Academic

Alumni

Academic Achievement - Staff

Academic Achievement - Faculty member

Academic Achievement-Student

B School Competition

B School Ranking

Campus Information and updates

Case Competition by B School

Case Competition by Corporate

Extra-Curricular

Entertainment and Events

Internationalization

News related to Education

Placement related information

Promotion of Programmes

Research Related Information

Startups Information

Mix Composite content and Others

Admissions

Academic

Alumni

Academic Achievement - Staff

Academic Achievement - Faculty member

Academic Achievement-Student

B School Competition
$0.211856^{*}$

$-0.001030$

$0.093062^{*}$

$-0.039529^{*}$

$0.088487^{*}$

$0.124694^{*}$

$0.069604^{*}$

$0.089473^{*}$

$0.046475^{*}$

$0.122998^{*}$

$-0.051852^{*}$

$-0.069725^{*}$

$-0.007616$

$0.100389^{*}$

$0.081273^{*}$

$0.127741^{*}$

$-0.058774^{*}$

$0.077959^{*}$

$0.115989^{*}$

$-0.023209^{*}$

$0.142131^{*}$

0.006586

$0.100678^{*}$

$-0.031913^{*}$

$0.096103^{*}$

$0.132310^{*}$

$0.077220^{*}$

$0.097089^{*}$

$0.054091^{*}$

$0.130614^{*}$

$-0.044236^{*}$

$-0.062109^{*}$

0.007616

$0.108005^{*}$

$0.088889^{*}$

$0.135357^{*}$

$-0.051158^{*}$

$0.085575^{*}$

$0.123605^{*}$

$-0.015593^{*}$

$0.149747^{*}$

$-0.101419^{*}$

$-0.007327$

$-0.139918^{*}$

$-0.011902^{*}$

$0.024305^{*}$

$-0.030785^{*}$

$-0.010916^{*}$

$-0.053914^{*}$

$0.022609^{*}$

$-0.152241^{*}$

$-0.170114^{*}$

$-0.100389^{*}$

$-0.108005^{*}$

$-0.019116^{*}$

$0.027352^{*}$

$-0.159163^{*}$

$-0.022430^{*}$

$0.015600^{*}$

$-0.123598^{*}$

$0.041742^{*}$

$-0.082303^{*}$

$0.011789^{*}$

$-0.120802^{*}$

0.007214

$0.043421^{*}$

$-0.011669^{*}$

$0.008200^{*}$
0.002244

0.002244

0.002244

0.002244

0.002244

0.002244

0.002244

0.002244

0.002244

0.002244

0.002244

0.002244

0.002244

0.002244

0.002244

0.002244

0.002244

0.002244

0.002244

0.002244

0.002244

0.002244

0.002244

0.002244

0.002244

0.002244

0.002244

0.002244

0.002244

0.002244

0.002244

0.002244

0.002244

0.002244

0.002244

0.002244

0.002244

0.002244

0.002244

0.002244

0.002244

0.002244

0.002244

0.002244

0.002244

0.002244

0.002244

0.002244

0.002244

0.002244

0.002244

0.002244

0.002244

0.002244

0.002244

0.002244

0.002244

0.002244

0.002244

0.002244

0.002244

0.002244

0.002244

0.002244

0.002244

0.002244

0.002244

0.002244
$0.000 \quad 0.20384$

$0.287-0.00905$

$0.000 \quad 0.08504$

$0.000 \quad-0.04755$

$0.000 \quad 0.08047$

$0.000 \quad 0.11667$

$0.000 \quad 0.06158$

$0.000 \quad 0.08145$

$0.000 \quad 0.03846$

$0.000 \quad 0.11498$

$0.000 \quad-0.05987$

$0.000 \quad-0.07774$

$0.088 \quad-0.01564$

$0.000 \quad 0.09237$

$0.000 \quad 0.07325$

$0.000 \quad 0.11972$

$0.000 \quad-0.06679$

$0.000 \quad 0.06994$

$0.000 \quad 0.10797$

$0.000 \quad-0.03123$

$0.083 \quad 0.13411$

$0.287 \quad-0.00143$

$0.000 \quad 0.09266$

$0.000-0.03993$

$0.000 \quad 0.08808$

$0.000 \quad 0.12429$

$0.000 \quad 0.06920$

$0.000 \quad 0.08907$

$0.000 \quad 0.04607$

$0.000 \quad 0.12259$

$0.000 \quad-0.05226$

$0.000 \quad-0.07013$

$0.088-0.00040$

$0.000 \quad 0.09999$

$0.000 \quad 0.08087$

$0.000 \quad 0.12734$

$0.000 \quad-0.05918$

$0.000 \quad 0.07756$

$0.000 \quad 0.11559$

$0.000 \quad-0.02361$

$0.000 \quad 0.14173$

$0.000 \quad-0.10944$

$0.127-0.01535$

$0.000 \quad-0.14794$

$0.000 \quad-0.01992$

$0.000 \quad 0.01629$

$0.000-0.03880$

$0.000-0.01894$

$0.000-0.06193$

$0.000 \quad 0.01459$

$0.000-0.16026$

$0.000-0.17813$

$0.000 \quad-0.10841$

$0.000-0.11602$

$0.000 \quad-0.02714$

$0.000 \quad 0.01933$

$0.000-0.16718$

$0.000-0.03045$

$0.000 \quad 0.00758$

$0.000-0.13162$

$0.000 \quad 0.03372$

$0.146-0.09032$

$0.000 \quad 0.00377$

$0.000 \quad-0.12882$

$0.146 \quad-0.00081$

$0.000 \quad 0.03540$

$0.000 \quad-0.01969$

0.038

0.21988

0.00699

0.10108

$-0.03151$

0.09651

0.13271

0.07762

0.09749

0.05449 
Vikram Kumar and Ramakrishnan Raman / Journal of Computer Science 2019, 15 (9): 1256.1282 DOI: 10.3844/jessp.2019.1256.1282

\begin{tabular}{|c|c|c|c|c|c|c|}
\hline \multirow{31}{*}{$\begin{array}{l}\text { News related to } \\
\text { education }\end{array}$} & B School Ranking & $-0.034798^{*}$ & 0.002244 & 0.000 & -0.04282 & -0.02678 \\
\hline & Campus Information and updates & $0.041725^{*}$ & 0.002244 & 0.000 & 0.03371 & 0.04974 \\
\hline & Case Competition by B School & $-0.133125^{*}$ & 0.002244 & 0.000 & -0.14114 & -0.12511 \\
\hline & Extra-Curricular & $-0.081273^{*}$ & 0.002244 & 0.000 & -0.08929 & -0.07325 \\
\hline & Entertainment and Events & $-0.088889^{*}$ & 0.002244 & 0.000 & -0.09691 & -0.08087 \\
\hline & Innovation & $0.019116^{*}$ & 0.002244 & 0.000 & 0.01110 & 0.02714 \\
\hline & News related to Education & $0.046468^{*}$ & 0.002244 & 0.000 & 0.03845 & 0.05449 \\
\hline & Placement related information & $-0.140047^{*}$ & 0.002244 & 0.000 & -0.14807 & -0.13203 \\
\hline & Promotion of Programmes & -0.003314 & 0.002244 & 0.997 & -0.01133 & 0.00471 \\
\hline & Research Related Information & $0.034716^{*}$ & 0.002244 & 0.000 & 0.02670 & 0.04274 \\
\hline & Startups Information & $-0.104482^{*}$ & 0.002244 & 0.000 & -0.11250 & -0.09646 \\
\hline & Mix Composite content and Others & $0.060858^{*}$ & 0.002244 & 0.000 & 0.05284 & 0.06888 \\
\hline & Admissions & $-0.128771^{*}$ & 0.002244 & 0.000 & -0.13679 & -0.12075 \\
\hline & Academic & $-0.034679^{*}$ & 0.002244 & 0.000 & -0.04270 & -0.02666 \\
\hline & Alumni & $-0.167270^{*}$ & 0.002244 & 0.000 & -0.17529 & -0.15925 \\
\hline & Academic Achievement - Faculty member & -0.003047 & 0.002244 & 0.992 & -0.01107 & 0.00497 \\
\hline & Academic Achievement- Student & $-0.058137^{*}$ & 0.002244 & 0.000 & -0.06616 & -0.05012 \\
\hline & B School Competition & $-0.038268^{*}$ & 0.002244 & 0.000 & -0.04629 & -0.03025 \\
\hline & B School Ranking & $-0.081266^{*}$ & 0.002244 & 0.000 & -0.08929 & -0.07325 \\
\hline & Campus Information and updates & -0.004743 & 0.002244 & 0.867 & -0.01276 & 0.00328 \\
\hline & Case Competition by B School & $-0.179593^{*}$ & 0.002244 & 0.000 & -0.18761 & -0.17157 \\
\hline & Case Competition by Corporate & $-0.197466^{*}$ & 0.002244 & 0.000 & -0.20549 & -0.18945 \\
\hline & Extra-Curricular & $-0.127741^{*}$ & 0.002244 & 0.000 & -0.13576 & -0.11972 \\
\hline & Entertainment and Events & $-0.135357^{*}$ & 0.002244 & 0.000 & -0.14338 & -0.12734 \\
\hline & Innovation & $-0.027352^{*}$ & 0.002244 & 0.000 & -0.03537 & -0.01933 \\
\hline & Internationalization & $-0.046468^{*}$ & 0.002244 & 0.000 & -0.05449 & -0.03845 \\
\hline & Placement related information & $-0.186515^{*}$ & 0.002244 & 0.000 & -0.19453 & -0.17850 \\
\hline & Promotion of Programmes & $-0.049782^{*}$ & 0.002244 & 0.000 & -0.05780 & -0.04176 \\
\hline & Research Related Information & $-0.011752^{*}$ & 0.002244 & 0.000 & -0.01977 & -0.00373 \\
\hline & Startups Information & $-0.150950^{*}$ & 0.002244 & 0.000 & -0.15897 & -0.14293 \\
\hline & Mix Composite content and Others & $0.014390^{*}$ & 0.002244 & 0.000 & 0.00637 & 0.02241 \\
\hline \multirow[t]{18}{*}{ information } & Alumni & $0.019245^{*}$ & 0.002244 & 0.000 & 0.01123 & 0.02726 \\
\hline & Academic Achievement - Staff & $0.147261^{*}$ & 0.002244 & 0.000 & 0.13924 & 0.15528 \\
\hline & Academic Achievement - Faculty member & $0.183468^{*}$ & 0.002244 & 0.000 & 0.17545 & 0.19149 \\
\hline & Academic Achievement- Student & $0.128378^{*}$ & 0.002244 & 0.000 & 0.12036 & 0.13640 \\
\hline & B School Competition & $0.148247^{*}$ & 0.002244 & 0.000 & 0.14023 & 0.15627 \\
\hline & B School Ranking & $0.105249^{*}$ & 0.002244 & 0.000 & 0.09723 & 0.11327 \\
\hline & Campus Information and updates & $0.181772^{*}$ & 0.002244 & 0.000 & 0.17375 & 0.18979 \\
\hline & Case Competition by B School & 0.006922 & 0.002244 & 0.203 & -0.00110 & 0.01494 \\
\hline & Case Competition by Corporate & $-0.010951^{*}$ & 0.002244 & 0.000 & -0.01897 & -0.00293 \\
\hline & Extra-Curricular & $0.058774^{*}$ & 0.002244 & 0.000 & 0.05075 & 0.06679 \\
\hline & Entertainment and Events & $0.051158^{*}$ & 0.002244 & 0.000 & 0.04314 & 0.05918 \\
\hline & Innovation & $0.159163^{*}$ & 0.002244 & 0.000 & 0.15114 & 0.16718 \\
\hline & Internationalization & $0.140047^{*}$ & 0.002244 & 0.000 & 0.13203 & 0.14807 \\
\hline & News related to Education & $0.186515^{*}$ & 0.002244 & 0.000 & 0.17850 & 0.19453 \\
\hline & Promotion of Programmes & $0.136733^{*}$ & 0.002244 & 0.000 & 0.12871 & 0.14475 \\
\hline & Research Related Information & $0.174763^{*}$ & 0.002244 & 0.000 & 0.16674 & 0.18278 \\
\hline & Startups Information & $0.035565^{*}$ & 0.002244 & 0.000 & 0.02755 & 0.04358 \\
\hline & Mix Composite content and Others & $0.200905^{*}$ & 0.002244 & 0.087 & 0.19289 & 0.20892 \\
\hline \multirow{13}{*}{$\begin{array}{l}\text { Promotion of } \\
\text { programmes }\end{array}$} & Admissions & $-0.078989^{*}$ & 0.002244 & 0.000 & -0.08701 & -0.07097 \\
\hline & Academic & $0.015103^{*}$ & 0.002244 & 0.000 & 0.00708 & 0.02312 \\
\hline & Alumni & $-0.117488^{*}$ & 0.002244 & 0.000 & -0.12551 & -0.10947 \\
\hline & Academic Achievement - Staff & $0.010528^{*}$ & 0.002244 & 0.001 & 0.00251 & 0.01855 \\
\hline & Academic Achievement - Faculty member & $0.046735^{*}$ & 0.002244 & 0.000 & 0.03872 & 0.05475 \\
\hline & Academic Achievement- Student & $-0.008355^{*}$ & 0.002244 & 0.030 & -0.01637 & -0.00034 \\
\hline & B School Competition & $0.011514^{*}$ & 0.002244 & 0.000 & 0.00349 & 0.01953 \\
\hline & B School Ranking & $-0.031484^{*}$ & 0.002244 & 0.000 & -0.03950 & -0.02346 \\
\hline & Campus Information and updates & $0.045039^{*}$ & 0.002244 & 0.000 & 0.03702 & 0.05306 \\
\hline & Case Competition by B School & $-0.129811^{*}$ & 0.002244 & 0.000 & -0.13783 & -0.12179 \\
\hline & Case Competition by Corporate & $-0.147684^{*}$ & 0.002244 & 0.000 & -0.15570 & -0.13966 \\
\hline & Extra-Curricular & $-0.077959^{*}$ & 0.002244 & 0.000 & -0.08598 & -0.06994 \\
\hline & Entertainment and Events & $-0.085575^{*}$ & 0.002244 & 0.000 & -0.09359 & -0.07756 \\
\hline
\end{tabular}


Vikram Kumar and Ramakrishnan Raman / Journal of Computer Science 2019, 15 (9): 1256.1282 DOI: 10.3844/jessp.2019.1256.1282

\begin{tabular}{|c|c|c|c|c|c|c|}
\hline \multirow{25}{*}{$\begin{array}{l}\text { Research related } \\
\text { information }\end{array}$} & Innovation & $0.022430^{*}$ & 0.002244 & 0.000 & 0.01441 & 0.03045 \\
\hline & Internationalization & 0.003314 & 0.002244 & 0.997 & -0.00471 & 0.01133 \\
\hline & News related to Education & $0.049782^{*}$ & 0.002244 & 0.000 & 0.04176 & 0.05780 \\
\hline & Research Related Information & $0.038030^{*}$ & 0.002244 & 0.000 & 0.03001 & 0.04605 \\
\hline & Startups Information & $-0.101168^{*}$ & 0.002244 & 0.000 & -0.10919 & -0.09315 \\
\hline & Mix Composite content and Others & $0.064172^{*}$ & 0.002244 & 0.000 & 0.05615 & 0.07219 \\
\hline & Admissions & $-0.117019^{*}$ & 0.002244 & 0.000 & -0.12504 & -0.10900 \\
\hline & Academic & $-0.022927^{*}$ & 0.002244 & 0.000 & -0.03095 & -0.01491 \\
\hline & Alumni & $-0.155518^{*}$ & 0.002244 & 0.000 & -0.16354 & -0.14750 \\
\hline & Academic Achievement - Staff & $-0.027502^{*}$ & 0.002244 & 0.000 & -0.03552 & -0.01948 \\
\hline & Academic Achievement - Faculty member & $0.008705^{*}$ & 0.002244 & 0.017 & 0.00069 & 0.01672 \\
\hline & Academic Achievement-Student & $-0.046385^{*}$ & 0.002244 & 0.000 & -0.05440 & -0.03837 \\
\hline & B School Competition & $-0.026516^{*}$ & 0.002244 & 0.000 & -0.03454 & -0.01850 \\
\hline & B School Ranking & $-0.069514^{*}$ & 0.002244 & 0.000 & -0.07753 & -0.06149 \\
\hline & Campus Information and updates & 0.007009 & 0.002244 & 0.185 & -0.00101 & 0.01503 \\
\hline & Case Competition by Corporate & $-0.185714^{*}$ & 0.002244 & 0.000 & -0.19373 & -0.17769 \\
\hline & Extra-Curricular & $-0.115989^{*}$ & 0.002244 & 0.000 & -0.12401 & -0.10797 \\
\hline & Entertainment and Events & $-0.123605^{*}$ & 0.002244 & 0.000 & -0.13162 & -0.11559 \\
\hline & Innovation & $-0.015600^{*}$ & 0.002244 & 0.000 & -0.02362 & -0.00758 \\
\hline & Internationalization & $-0.034716^{*}$ & 0.002244 & 0.000 & -0.04274 & -0.02670 \\
\hline & News related to Education & $0.011752^{*}$ & 0.002244 & 0.000 & 0.00373 & 0.01977 \\
\hline & Placement related information & $-0.174763^{*}$ & 0.002244 & 0.000 & -0.18278 & -0.16674 \\
\hline & Promotion of Programmes & $-0.038030^{*}$ & 0.002244 & 0.000 & -0.04605 & -0.03001 \\
\hline & Startups Information & $-0.139198^{*}$ & 0.002244 & 0.000 & -0.14722 & -0.13118 \\
\hline & Mix Composite content and Others & $0.026142^{*}$ & 0.002244 & 0.000 & 0.01812 & 0.03416 \\
\hline \multirow[t]{18}{*}{ Startups information } & Admissions & $0.022179^{*}$ & 0.002244 & 0.000 & 0.01416 & 0.03020 \\
\hline & Academic & $0.116271^{*}$ & 0.002244 & 0.000 & 0.10825 & 0.12429 \\
\hline & Alumni & $-0.016320^{*}$ & 0.002244 & 0.000 & -0.02434 & -0.00830 \\
\hline & Academic Achievement - Staff & $0.111696^{*}$ & 0.002244 & 0.000 & 0.10368 & 0.11972 \\
\hline & Academic Achievement - Faculty member & $0.147903^{*}$ & 0.002244 & 0.000 & 0.13988 & 0.15592 \\
\hline & Academic Achievement- Student & $0.092813^{*}$ & 0.002244 & 0.000 & 0.08479 & 0.10083 \\
\hline & B School Competition & $0.112682^{*}$ & 0.002244 & 0.000 & 0.10466 & 0.12070 \\
\hline & Case Competition by B School & $-0.028643^{*}$ & 0.002244 & 0.000 & -0.03666 & -0.02062 \\
\hline & Case Competition by Corporate & $-0.046516^{*}$ & 0.002244 & 0.000 & -0.05454 & -0.03850 \\
\hline & Extra-Curricular & $0.023209^{*}$ & 0.002244 & 0.000 & 0.01519 & 0.03123 \\
\hline & Entertainment and Events & $0.015593^{*}$ & 0.002244 & 0.000 & 0.00757 & 0.02361 \\
\hline & Innovation & $0.123598^{*}$ & 0.002244 & 0.000 & 0.11558 & 0.13162 \\
\hline & Internationalization & $0.104482^{*}$ & 0.002244 & 0.000 & 0.09646 & 0.11250 \\
\hline & News related to Education & $0.150950^{*}$ & 0.002244 & 0.000 & 0.14293 & 0.15897 \\
\hline & Placement related information & $-0.035565^{*}$ & 0.002244 & 0.000 & -0.04358 & -0.02755 \\
\hline & Promotion of Programmes & $0.101168^{*}$ & 0.002244 & 0.000 & 0.09315 & 0.10919 \\
\hline & Research Related Information & $0.139198^{*}$ & 0.002244 & 0.000 & 0.13118 & 0.14722 \\
\hline & Mix Composite content and Others & $0.165340^{*}$ & 0.002244 & 0.000 & 0.15732 & 0.17336 \\
\hline \multirow{20}{*}{$\begin{array}{l}\text { Mix composite } \\
\text { content and others }\end{array}$} & Admissions & $-0.143161^{*}$ & 0.002244 & 0.000 & -0.15118 & -0.13514 \\
\hline & Academic & $-0.049069^{*}$ & 0.002244 & 0.000 & -0.05709 & -0.04105 \\
\hline & Alumni & $-0.181660^{*}$ & 0.002244 & 0.000 & -0.18968 & -0.17364 \\
\hline & Academic Achievement - Staff & $-0.053644^{*}$ & 0.002244 & 0.000 & -0.06166 & -0.04562 \\
\hline & Academic Achievement - Faculty member & $-0.017437^{*}$ & 0.002244 & 0.000 & -0.02546 & -0.00942 \\
\hline & Academic Achievement-Student & $-0.072527^{*}$ & 0.002244 & 0.000 & -0.08055 & -0.06451 \\
\hline & B School Competition & $-0.052658^{*}$ & 0.002244 & 0.060 & -0.06068 & -0.04464 \\
\hline & B School Ranking & $-0.095656^{*}$ & 0.002244 & 0.000 & -0.10368 & -0.08764 \\
\hline & Campus Information and updates & $-0.019133^{*}$ & 0.002244 & 0.000 & -0.02715 & -0.01111 \\
\hline & Case Competition by B School & $-0.193983^{*}$ & 0.002244 & 0.000 & -0.20200 & -0.18596 \\
\hline & Case Competition by Corporate & $-0.211856^{*}$ & 0.002244 & 0.000 & -0.21988 & -0.20384 \\
\hline & Extra-Curricular & $-0.142131^{*}$ & 0.002244 & 0.083 & -0.15015 & -0.13411 \\
\hline & Entertainment and Events & $-0.149747^{*}$ & 0.002244 & 0.000 & -0.15777 & -0.14173 \\
\hline & Innovation & $-0.041742^{*}$ & 0.002244 & 0.000 & -0.04976 & -0.03372 \\
\hline & Internationalization & $-0.060858^{*}$ & 0.002244 & 0.000 & -0.06888 & -0.05284 \\
\hline & News related to Education & $-0.014390^{*}$ & 0.002244 & 0.000 & -0.02241 & -0.00637 \\
\hline & Placement related information & $-0.200905^{*}$ & 0.002244 & 0.087 & -0.20892 & -0.19289 \\
\hline & Promotion of Programmes & $-0.064172^{*}$ & 0.002244 & 0.000 & -0.07219 & -0.05615 \\
\hline & Research Related Information & $-0.026142^{*}$ & 0.002244 & 0.000 & -0.03416 & -0.01812 \\
\hline & Startups Information & $-0.165340^{*}$ & 0.002244 & 0.000 & -0.17336 & -0.15732 \\
\hline
\end{tabular}

*. The mean difference is significant at the 0.05 level. 
Vikram Kumar and Ramakrishnan Raman / Journal of Computer Science 2019, 15 (9): 1256.1282 DOI: 10.3844/jessp.2019.1256.1282

Table 8: No significant difference in proportional engagement for posts - category

Categories

Admissions

Academic

Alumni

Academic Achievement - Staff

Academic Achievement - Faculty member

Academic Achievement- Student

B School Competition

B School Ranking

Campus Information and updates

Case Competition by B School

Case Competition by Corporate

Extra-Curricular

Entertainment and Events

Innovation

Internationalization

News related to Education

Placement related information

Promotion of Programmes

Research Related Information

Startups Information

Mix Composite content and Others
Statistically NO significant difference in proportional engagement in posts posted in the following categories Extra-Curricular $(p=0.287)$

Entertainment and Events $(\mathrm{p}=0.287)$

Academic Achievement - Staff $(p=0.901)$

B School Competition $(\mathrm{p}=0.992)$

Innovation $(\mathrm{p}=0.127)$

NIL

Academic $(\mathrm{p}=0.901)$

B School Competition $(p=0.992)$

Internationalization $(\mathrm{p}=0.146)$

Campus Information and updates

News related to Education $(\mathrm{p}=0.992)$

NIL

Academic $(p=0.992)$

Academic Achievement - Staff $(\mathrm{p}=0.992)$

Internationalization $(\mathrm{p}=0.038)$

Mix Composite content and Others $(\mathrm{p}=0.060)$

NIL

Academic Achievement - Faculty member $(\mathrm{p}=0.877)$

News related to Education $(\mathrm{p}=0.867)$

Research Related Information $(p=0.185)$

Placement related information $(\mathrm{p}=0.203)$

NIL

Admissions $(p=0.287)$

Entertainment and Events $(\mathrm{p}=0.088)$

Mix Composite content and Others $(\mathrm{p}=0.083)$

Admissions $(\mathrm{p}=0.287)$

Extra-Curricular $(\mathrm{p}=0.088)$

Academic $(\mathrm{p}=0.127)$

Academic Achievement - Staff $(p=0.146)$

Promotion of Programmes $(p=0.997)$

B School Competition $(\mathrm{p}=0.038)$

Academic Achievement - Faculty member $(p=0.992)$

Campus Information and updates $(\mathrm{p}=0.867)$

Case Competition by B School $(p=0.203$ )

Mix Composite content and Others $(\mathrm{p}=0.087)$

Internationalization $(\mathrm{p}=0.997)$

Campus Information and updates $(\mathrm{p}=0.185)$

NIL

B School Competition $(\mathrm{p}=0.060)$

Extra-Curricular $(\mathrm{p}=0.083)$

Placement related information $(\mathrm{p}=0.087)$

Table 9: Descriptive information of format type of post

\begin{tabular}{|c|c|c|c|c|c|c|c|c|}
\hline \multirow[b]{2}{*}{ Format Type } & \multirow[b]{2}{*}{$\mathrm{N}$} & \multirow[b]{2}{*}{ Mean } & \multirow[b]{2}{*}{$\begin{array}{l}\text { Std. } \\
\text { Deviation }\end{array}$} & \multirow[b]{2}{*}{$\begin{array}{l}\text { Std. } \\
\text { Error }\end{array}$} & \multicolumn{2}{|c|}{$95 \%$ confidence interval for mean } & \multirow[b]{2}{*}{ Min } & \multirow[b]{2}{*}{$\operatorname{Max}$} \\
\hline & & & & & Lower bound & Upper bound & & \\
\hline Blog Post link & 100 & 0.001892 & 0.0005626 & 0.0000563 & 0.00178 & 0.002004 & 0.001 & 0.0029 \\
\hline Call for action & 100 & 0.231436 & 0.0008309 & 0.0000831 & 0.231271 & 0.231601 & 0.2301 & 0.233 \\
\hline Info-graphic & 100 & 0.193517 & 0.0007145 & 0.0000715 & 0.193375 & 0.193659 & 0.1922 & 0.1947 \\
\hline Link to URL & 100 & 0.012175 & 0.0039776 & 0.0003978 & 0.011386 & 0.012965 & 0.0011 & 0.0148 \\
\hline Message & 100 & 0.067973 & 0.025063 & 0.0025063 & 0.063 & 0.072946 & 0.0586 & 0.1389 \\
\hline Picture & 100 & 0.580846 & 0.2023717 & 0.0202372 & 0.540691 & 0.621001 & 0.0081 & 0.653 \\
\hline Picture and Text & 100 & 0.625804 & 0.2085466 & 0.0208547 & 0.584424 & 0.667184 & 0.0356 & 0.6999 \\
\hline Podcast & 100 & 0.016775 & 0.0236797 & 0.002368 & 0.012076 & 0.021473 & 0.007 & 0.0838 \\
\hline Question & 100 & 0.166476 & 0.0792777 & 0.0079278 & 0.150746 & 0.182207 & 0.1375 & 0.3908 \\
\hline User Generated Content & 100 & 0.395198 & 0.0086743 & 0.0008674 & 0.393477 & 0.396919 & 0.3903 & 0.4196 \\
\hline User Generated Content & 100 & 0.340861 & 0.1183612 & 0.0118361 & 0.317375 & 0.364346 & 0.0059 & 0.3833 \\
\hline Video and Text & 100 & 0.270169 & 0.0659535 & 0.0065954 & 0.257083 & 0.283256 & 0.0835 & 0.2945 \\
\hline Mix Mode and Others & 100 & 0.027199 & 0.0735178 & 0.0073518 & 0.012611 & 0.041786 & 0.0003 & 0.2353 \\
\hline
\end{tabular}


The Hypothesis (B) $H_{0}$ - There is no significant difference in proportional engagement for format of the post was crafted to check if there was any significant difference in the engagement and its relation the format of the post. The descriptive statistical information given in Table 9 gives descriptive statistical information, including mean, standard deviation and $95 \%$ confidence intervals for the dependent variable- proportional engagement score- for each format of the Facebook post where all the 14 formats have been listed. Table10 is the output of the ANOVA analysis which is used to test the Hypothesis (B) to understand if there is a statistically significant difference between the group means of proportional engagement scores and the format of the post. It can be inferred from the significance value 0.000 (i.e., $\mathrm{p}=0.000$ ), which is below 0.05 , that there is a statistically significant difference between in proportional engagement and he format of the post. Which clearly indicates that different posts under different format have different engagements. To know which of those specific format differed in the engagement, Tukey post hoc test was used and the Table 11 gives the output which is the multiple comparisons table for the Format type. Multiple comparisons Table 11 it can be statistically inferred that there is a statistically significant difference in proportional engagement in posts that were posted in Blog Post Link format in comparison with all other formats $(\mathrm{p}=$ 0.000), but there is a statistically no significant difference between the posts of Blog Post Link format and the posts under Link to URL $(p=0.932)$ and Podcast format $(\mathrm{p}=0.997)$. For the posts of the format 'User Generated Content' and 'Video' it can be statistically inferred that there is a statistically significant difference in proportional engagement in posts that were posted in comparison with all other formats $(p=0.000)$. The Table 12 gives the details for all the formats for which there is a statistically no significant difference in proportional engagement in posts when compared to other formats.

Table 10: ANOVA analysis - format type

Format type engagement

\begin{tabular}{llllll}
\hline & Sum of Squares & df & Mean square & F & Sig. \\
\hline Between groups & 54.573 & 13 & 4.198 & 507.393 \\
Within groups & 11.467 & 1386 & 0.008 & \\
Total & 66.040 & 1399 & & \\
\hline
\end{tabular}

Table 11: Multiple comparisons of format type

Dependent Variable: Engagement

Tukey HSD

\begin{tabular}{|c|c|c|c|c|c|c|}
\hline \multirow[b]{2}{*}{ (I) Format } & \multirow[b]{2}{*}{ (J) Format } & \multirow{2}{*}{$\begin{array}{l}\text { Mean } \\
\text { difference (I-J) }\end{array}$} & \multirow[b]{2}{*}{ Std. Error } & \multirow[b]{2}{*}{ Sig. } & \multicolumn{2}{|c|}{ 95\% Confidence interval } \\
\hline & & & & & Lower bound & Upper bound \\
\hline \multirow[t]{13}{*}{ Blog Post link } & Call for action & $-0.2295440^{*}$ & 0.0128635 & 0.000 & -0.272761 & -0.186327 \\
\hline & Info-graphic & $-0.1916250^{*}$ & 0.0128635 & 0.000 & -0.234842 & -0.148408 \\
\hline & Link to URL & -0.0102834 & 0.0128635 & 0.932 & -0.053500 & 0.032933 \\
\hline & Message & $-0.0660810^{*}$ & 0.0128635 & 0.070 & -0.109298 & -0.022864 \\
\hline & Opinion & $-0.1345080^{*}$ & 0.0128635 & 0.000 & -0.177725 & -0.091291 \\
\hline & Picture & $-0.5789540^{*}$ & 0.0128635 & 0.000 & -0.622171 & -0.535737 \\
\hline & Picture and Text & $-0.6239118^{*}$ & 0.0128635 & 0.000 & -0.667129 & -0.580695 \\
\hline & Podcast & -0.0148826 & 0.0128635 & 0.997 & -0.058099 & 0.028334 \\
\hline & Question & $-0.1645844^{*}$ & 0.0128635 & 0.000 & -0.207801 & -0.121368 \\
\hline & User Generated Content & $-0.3933058^{*}$ & 0.0128635 & 0.000 & -0.436523 & -0.350089 \\
\hline & Video & $-0.3389688^{*}$ & 0.0128635 & 0.000 & -0.382186 & -0.295752 \\
\hline & Video and Text & $-0.2682772^{*}$ & 0.0128635 & 0.000 & -0.311494 & -0.225060 \\
\hline & Mix Mode and Others & -0.0253066 & 0.0128635 & 0.788 & -0.068523 & 0.017910 \\
\hline \multirow[t]{13}{*}{ Call for action } & Blog Post link & $0.2295440^{*}$ & 0.0128635 & 0.000 & 0.186327 & 0.272761 \\
\hline & Info-graphic & 0.0379190 & 0.0128635 & 0.159 & -0.005298 & 0.081136 \\
\hline & Link to URL & $0.2192606^{*}$ & 0.0128635 & 0.000 & 0.176044 & 0.262477 \\
\hline & Message & $0.1634630^{*}$ & 0.0128635 & 0.000 & 0.120246 & 0.206680 \\
\hline & Opinion & $0.0950360^{*}$ & 0.0128635 & 0.000 & 0.051819 & 0.138253 \\
\hline & Picture & $-0.3494100^{*}$ & 0.0128635 & 0.000 & -0.392627 & -0.306193 \\
\hline & Picture and Text & $-0.3943678^{*}$ & 0.0128635 & 0.000 & -0.437585 & -0.351151 \\
\hline & Podcast & $0.2146614^{*}$ & 0.0128635 & 0.000 & 0.171445 & 0.257878 \\
\hline & Question & $0.0649596^{*}$ & 0.0128635 & 0.000 & 0.021743 & 0.108176 \\
\hline & User Generated Content & $-0.1637618^{*}$ & 0.0128635 & 0.000 & -0.206979 & -0.120545 \\
\hline & Video & $-0.1094248^{*}$ & 0.0128635 & 0.000 & -0.152642 & -0.066208 \\
\hline & Video and Text & -0.0387332 & 0.0128635 & 0.135 & -0.081950 & 0.004484 \\
\hline & Mix Mode and Others & $0.2042374^{*}$ & 0.0128635 & 0.000 & 0.161021 & 0.247454 \\
\hline
\end{tabular}


Vikram Kumar and Ramakrishnan Raman / Journal of Computer Science 2019, 15 (9): 1256.1282 DOI: 10.3844/jessp.2019.1256.1282

Table 11: Continue

\begin{tabular}{|c|c|c|c|c|c|c|}
\hline \multirow[t]{13}{*}{ Info-graphic } & Blog Post link & $0.1916250^{*}$ & 0.0128635 & 0.000 & 0.148408 & 0.234842 \\
\hline & Call for action & -0.0379190 & 0.0128635 & 0.159 & -0.081136 & 0.005298 \\
\hline & Link to URL & $0.1813416^{*}$ & 0.0128635 & 0.000 & 0.138125 & 0.224558 \\
\hline & Message & $0.1255440^{*}$ & 0.0128635 & 0.000 & 0.082327 & 0.168761 \\
\hline & Opinion & $0.0571170^{*}$ & 0.0128635 & 0.001 & 0.013900 & 0.100334 \\
\hline & Picture & $-0.3873290^{*}$ & 0.0128635 & 0.000 & -0.430546 & -0.344112 \\
\hline & Picture and Text & $-0.4322868^{*}$ & 0.0128635 & 0.000 & -0.475504 & -0.389070 \\
\hline & Podcast & $0.1767424^{*}$ & 0.0128635 & 0.000 & 0.133526 & 0.219959 \\
\hline & Question & 0.0270406 & 0.0128635 & 0.700 & -0.016176 & 0.070257 \\
\hline & User Generated Content & $-0.2016808^{*}$ & 0.0128635 & 0.000 & -0.244898 & -0.158464 \\
\hline & Video & $-0.1473438^{*}$ & 0.0128635 & 0.000 & -0.190561 & -0.104127 \\
\hline & Video and Text & $-0.0766522^{*}$ & 0.0128635 & 0.000 & -0.119869 & -0.033435 \\
\hline & Mix Mode and Others & $0.1663184^{*}$ & 0.0128635 & 0.000 & 0.123102 & 0.209535 \\
\hline \multirow[t]{13}{*}{ Link to URL } & Blog Post link & 0.0102834 & 0.0128635 & 0.932 & -0.032933 & 0.053500 \\
\hline & Call for action & $-0.2192606^{*}$ & 0.0128635 & 0.000 & -0.262477 & -0.176044 \\
\hline & Info-graphic & $-0.1813416^{*}$ & 0.0128635 & 0.000 & -0.224558 & -0.138125 \\
\hline & Message & $-0.0557976^{*}$ & 0.0128635 & 0.081 & -0.099014 & -0.012581 \\
\hline & Opinion & $-0.1242246^{*}$ & 0.0128635 & 0.000 & -0.167441 & -0.081008 \\
\hline & Picture & $-0.5686706^{*}$ & 0.0128635 & 0.000 & -0.611887 & -0.525454 \\
\hline & Picture and Text & $-0.6136284^{*}$ & 0.0128635 & 0.000 & -0.656845 & -0.570412 \\
\hline & Podcast & -0.0045992 & 0.0128635 & 0.932 & -0.047816 & 0.038618 \\
\hline & Question & $-0.1543010^{*}$ & 0.0128635 & 0.000 & -0.197518 & -0.111084 \\
\hline & User Generated Content & $-0.3830224^{*}$ & 0.0128635 & 0.000 & -0.426239 & -0.339806 \\
\hline & Video & $-0.3286854^{*}$ & 0.0128635 & 0.000 & -0.371902 & -0.285469 \\
\hline & Video and Text & $-0.2579938^{*}$ & 0.0128635 & 0.000 & -0.301211 & -0.214777 \\
\hline & Mix Mode and Others & -0.0150232 & 0.0128635 & 0.997 & -0.058240 & 0.028194 \\
\hline \multirow[t]{13}{*}{ Message } & Blog Post link & $0.0660810^{*}$ & 0.0128635 & 0.070 & 0.022864 & 0.109298 \\
\hline & Call for action & $-0.1634630^{*}$ & 0.0128635 & 0.000 & -0.206680 & -0.120246 \\
\hline & Info-graphic & $-0.1255440^{*}$ & 0.0128635 & 0.000 & -0.168761 & -0.082327 \\
\hline & Link to URL & $0.0557976^{*}$ & 0.0128635 & 0.081 & 0.012581 & 0.099014 \\
\hline & Opinion & $-0.0684270^{*}$ & 0.0128635 & 0.000 & -0.111644 & -0.025210 \\
\hline & Picture & $-0.5128730^{*}$ & 0.0128635 & 0.000 & -0.556090 & -0.469656 \\
\hline & Picture and Text & $-0.5578308^{*}$ & 0.0128635 & 0.000 & -0.601048 & -0.514614 \\
\hline & Podcast & $0.0511984^{*}$ & 0.0128635 & 0.060 & 0.007982 & 0.094415 \\
\hline & Question & $-0.0985034^{*}$ & 0.0128635 & 0.000 & -0.141720 & -0.055287 \\
\hline & User Generated Content & $-0.3272248^{*}$ & 0.0128635 & 0.000 & -0.370442 & -0.284008 \\
\hline & Video & $-0.2728878^{*}$ & 0.0128635 & 0.000 & -0.316105 & -0.229671 \\
\hline & Video and Text & $-0.2021962^{*}$ & 0.0128635 & 0.000 & -0.245413 & -0.158979 \\
\hline & Mix Mode and Others & 0.0407744 & 0.0128635 & 0.088 & -0.002442 & 0.083991 \\
\hline \multirow[t]{13}{*}{ Opinion } & Blog Post link & $0.1345080^{*}$ & 0.0128635 & 0.000 & 0.091291 & 0.177725 \\
\hline & Call for action & $-0.0950360^{*}$ & 0.0128635 & 0.000 & -0.138253 & -0.051819 \\
\hline & Info-graphic & $-0.0571170^{*}$ & 0.0128635 & 0.001 & -0.100334 & -0.013900 \\
\hline & Link to URL & $0.1242246^{*}$ & 0.0128635 & 0.000 & 0.081008 & 0.167441 \\
\hline & Message & $0.0684270^{*}$ & 0.0128635 & 0.000 & 0.025210 & 0.111644 \\
\hline & Picture & $-0.4444460^{*}$ & 0.0128635 & 0.000 & -0.487663 & -0.401229 \\
\hline & Picture and Text & $-0.4894038^{*}$ & 0.0128635 & 0.000 & -0.532621 & -0.446187 \\
\hline & Podcast & $0.1196254^{*}$ & 0.0128635 & 0.000 & 0.076409 & 0.162842 \\
\hline & Question & -0.0300764 & 0.0128635 & 0.527 & -0.073293 & 0.013140 \\
\hline & User Generated Content & $-0.2587978^{*}$ & 0.0128635 & 0.000 & -0.302015 & -0.215581 \\
\hline & Video & $-0.2044608^{*}$ & 0.0128635 & 0.000 & -0.247678 & -0.161244 \\
\hline & Video and Text & $-0.1337692^{*}$ & 0.0128635 & 0.000 & -0.176986 & -0.090552 \\
\hline & Mix Mode and Others & $0.1092014^{*}$ & 0.0128635 & 0.000 & 0.065985 & 0.152418 \\
\hline \multirow[t]{11}{*}{ Picture } & Blog Post link & $0.5789540^{*}$ & 0.0128635 & 0.000 & 0.535737 & 0.622171 \\
\hline & Call for action & $0.3494100^{*}$ & 0.0128635 & 0.000 & 0.306193 & 0.392627 \\
\hline & Info-graphic & $0.3873290^{*}$ & 0.0128635 & 0.000 & 0.344112 & 0.430546 \\
\hline & Link to URL & $0.5686706^{*}$ & 0.0128635 & 0.000 & 0.525454 & 0.611887 \\
\hline & Message & $0.5128730^{*}$ & 0.0128635 & 0.000 & 0.469656 & 0.556090 \\
\hline & Opinion & $0.4444460^{*}$ & 0.0128635 & 0.000 & 0.401229 & 0.487663 \\
\hline & Picture and Text & $-0.0449578^{*}$ & 0.0128635 & 0.032 & -0.088175 & -0.001741 \\
\hline & Podcast & $0.5640714^{*}$ & 0.0128635 & 0.000 & 0.520855 & 0.607288 \\
\hline & Question & $0.4143696^{*}$ & 0.0128635 & 0.000 & 0.371153 & 0.457586 \\
\hline & User Generated Content & $0.1856482^{*}$ & 0.0128635 & 0.000 & 0.142431 & 0.228865 \\
\hline & Video & $0.2399852^{*}$ & 0.0128635 & 0.000 & 0.196768 & 0.283202 \\
\hline
\end{tabular}


Vikram Kumar and Ramakrishnan Raman / Journal of Computer Science 2019, 15 (9): 1256.1282 DOI: 10.3844/jessp.2019.1256.1282

Table 11: Continue

\begin{tabular}{|c|c|c|c|c|c|c|}
\hline & Video and Text & $0.3106768^{*}$ & 0.0128635 & 0.000 & 0.267460 & 0.353894 \\
\hline & Mix Mode and Others & $0.5536474^{*}$ & 0.0128635 & 0.000 & 0.510431 & 0.596864 \\
\hline \multirow[t]{13}{*}{ Picture and Text } & Blog Post link & $0.6239118^{*}$ & 0.0128635 & 0.000 & 0.580695 & 0.667129 \\
\hline & Call for action & $0.3943678^{*}$ & 0.0128635 & 0.000 & 0.351151 & 0.437585 \\
\hline & Info-graphic & $0.4322868^{*}$ & 0.0128635 & 0.000 & 0.389070 & 0.475504 \\
\hline & Link to URL & $0.6136284^{*}$ & 0.0128635 & 0.000 & 0.570412 & 0.656845 \\
\hline & Message & $0.5578308^{*}$ & 0.0128635 & 0.000 & 0.514614 & 0.601048 \\
\hline & Opinion & $0.4894038^{*}$ & 0.0128635 & 0.000 & 0.446187 & 0.532621 \\
\hline & Picture & $0.0449578^{*}$ & 0.0128635 & 0.032 & 0.001741 & 0.088175 \\
\hline & Podcast & $0.6090292^{*}$ & 0.0128635 & 0.000 & 0.565812 & 0.652246 \\
\hline & Question & $0.4593274^{*}$ & 0.0128635 & 0.000 & 0.416111 & 0.502544 \\
\hline & User Generated Content & $0.2306060^{*}$ & 0.0128635 & 0.000 & 0.187389 & 0.273823 \\
\hline & Video & $0.2849430^{*}$ & 0.0128635 & 0.000 & 0.241726 & 0.328160 \\
\hline & Video and Text & $0.3556346^{*}$ & 0.0128635 & 0.000 & 0.312418 & 0.398851 \\
\hline & Mix Mode and Others & $0.5986052^{*}$ & 0.0128635 & 0.000 & 0.555388 & 0.641822 \\
\hline \multirow[t]{13}{*}{ Podcast } & Blog Post link & 0.0148826 & 0.0128635 & 0.997 & -0.028334 & 0.058099 \\
\hline & Call for action & $-0.2146614^{*}$ & 0.0128635 & 0.000 & -0.257878 & -0.171445 \\
\hline & Info-graphic & $-0.1767424^{*}$ & 0.0128635 & 0.000 & -0.219959 & -0.133526 \\
\hline & Link to URL & 0.0045992 & 0.0128635 & 0.932 & -0.038618 & 0.047816 \\
\hline & Message & $-0.0511984^{*}$ & 0.0128635 & 0.060 & -0.094415 & -0.007982 \\
\hline & Opinion & $-0.1196254^{*}$ & 0.0128635 & 0.000 & -0.162842 & -0.076409 \\
\hline & Picture & $-0.5640714^{*}$ & 0.0128635 & 0.000 & -0.607288 & -0.520855 \\
\hline & Picture and Text & $-0.6090292^{*}$ & 0.0128635 & 0.000 & -0.652246 & -0.565812 \\
\hline & Question & $-0.1497018^{*}$ & 0.0128635 & 0.000 & -0.192919 & -0.106485 \\
\hline & User Generated Content & $-0.3784232^{*}$ & 0.0128635 & 0.000 & -0.421640 & -0.335206 \\
\hline & Video & $-0.3240862^{*}$ & 0.0128635 & 0.000 & -0.367303 & -0.280869 \\
\hline & Video and Text & $-0.2533946^{*}$ & 0.0128635 & 0.000 & -0.296611 & -0.210178 \\
\hline & Mix Mode and Others & -0.0104240 & 0.0128635 & 0.912 & -0.053641 & 0.032793 \\
\hline \multirow[t]{13}{*}{ Question } & Blog Post link & $0.1645844^{*}$ & 0.0128635 & 0.000 & 0.121368 & 0.207801 \\
\hline & Call for action & $-0.0649596^{*}$ & 0.0128635 & 0.000 & -0.108176 & -0.021743 \\
\hline & Info-graphic & -0.0270406 & 0.0128635 & 0.700 & -0.070257 & 0.016176 \\
\hline & Link to URL & $0.1543010^{*}$ & 0.0128635 & 0.000 & 0.111084 & 0.197518 \\
\hline & Message & $0.0985034^{*}$ & 0.0128635 & 0.000 & 0.055287 & 0.141720 \\
\hline & Opinion & 0.0300764 & 0.0128635 & 0.527 & -0.013140 & 0.073293 \\
\hline & Picture & $-0.4143696^{*}$ & 0.0128635 & 0.000 & -0.457586 & -0.371153 \\
\hline & Picture and Text & $-0.4593274^{*}$ & 0.0128635 & 0.000 & -0.502544 & -0.416111 \\
\hline & Podcast & $0.1497018^{*}$ & 0.0128635 & 0.000 & 0.106485 & 0.192919 \\
\hline & User Generated Content & $-0.2287214^{*}$ & 0.0128635 & 0.000 & -0.271938 & -0.185505 \\
\hline & Video & $-0.1743844^{*}$ & 0.0128635 & 0.000 & -0.217601 & -0.131168 \\
\hline & Video and Text & $-0.1036928^{*}$ & 0.0128635 & 0.000 & -0.146910 & -0.060476 \\
\hline & Mix Mode and Others & $0.1392778^{*}$ & 0.0128635 & 0.000 & 0.096061 & 0.182495 \\
\hline \multirow[t]{13}{*}{ User Generated Content } & Blog Post link & $0.3933058^{*}$ & 0.0128635 & 0.000 & 0.350089 & 0.436523 \\
\hline & Call for action & $0.1637618^{*}$ & 0.0128635 & 0.000 & 0.120545 & 0.206979 \\
\hline & Info-graphic & $0.2016808^{*}$ & 0.0128635 & 0.000 & 0.158464 & 0.244898 \\
\hline & Link to URL & $0.3830224^{*}$ & 0.0128635 & 0.000 & 0.339806 & 0.426239 \\
\hline & Message & $0.3272248^{*}$ & 0.0128635 & 0.000 & 0.284008 & 0.370442 \\
\hline & Opinion & $0.2587978^{*}$ & 0.0128635 & 0.000 & 0.215581 & 0.302015 \\
\hline & Picture & $-0.1856482^{*}$ & 0.0128635 & 0.000 & -0.228865 & -0.142431 \\
\hline & Picture and Text & $-0.2306060^{*}$ & 0.0128635 & 0.000 & -0.273823 & -0.187389 \\
\hline & Podcast & $0.3784232^{*}$ & 0.0128635 & 0.000 & 0.335206 & 0.421640 \\
\hline & Question & $0.2287214^{*}$ & 0.0128635 & 0.000 & 0.185505 & 0.271938 \\
\hline & Video & $0.0543370^{*}$ & 0.0128635 & 0.002 & 0.011120 & 0.097554 \\
\hline & Video and Text & $0.1250286^{*}$ & 0.0128635 & 0.000 & 0.081812 & 0.168245 \\
\hline & Mix Mode and Others & $0.3679992^{*}$ & 0.0128635 & 0.000 & 0.324782 & 0.411216 \\
\hline \multirow{11}{*}{ Video } & Blog Post link & $0.3389688^{*}$ & 0.0128635 & 0.000 & 0.295752 & 0.382186 \\
\hline & Call for action & $0.1094248^{*}$ & 0.0128635 & 0.000 & 0.066208 & 0.152642 \\
\hline & Info-graphic & $0.1473438^{*}$ & 0.0128635 & 0.000 & 0.104127 & 0.190561 \\
\hline & Link to URL & $0.3286854^{*}$ & 0.0128635 & 0.000 & 0.285469 & 0.371902 \\
\hline & Message & $0.2728878^{*}$ & 0.0128635 & 0.000 & 0.229671 & 0.316105 \\
\hline & Opinion & $0.2044608^{*}$ & 0.0128635 & 0.000 & 0.161244 & 0.247678 \\
\hline & Picture & $-0.2399852^{*}$ & 0.0128635 & 0.000 & -0.283202 & -0.196768 \\
\hline & Picture and Text & $-0.2849430^{*}$ & 0.0128635 & 0.000 & -0.328160 & -0.241726 \\
\hline & Podcast & $0.3240862^{*}$ & 0.0128635 & 0.000 & 0.280869 & 0.367303 \\
\hline & Question & $0.1743844^{*}$ & 0.0128635 & 0.000 & 0.131168 & 0.217601 \\
\hline & User Generated Content & $-0.0543370^{*}$ & 0.0128635 & 0.002 & -0.097554 & -0.011120 \\
\hline
\end{tabular}


Vikram Kumar and Ramakrishnan Raman / Journal of Computer Science 2019, 15 (9): 1256.1282 DOI: 10.3844/jessp.2019.1256.1282

Table 11: Continue

\begin{tabular}{lllllll} 
Table 11: Continue & & & & \\
& Video and Text & $0.0706916^{*}$ & 0.0128635 & 0.000 & 0.027475 & 0.113908 \\
Video and Text & Mix Mode and Others & $0.3136622^{*}$ & 0.0128635 & 0.000 & 0.270445 & 0.356879 \\
& Blog Post link & $0.2682772^{*}$ & 0.0128635 & 0.000 & 0.225060 & 0.311494 \\
& Call for action & 0.0387332 & 0.0128635 & 0.135 & -0.004484 & 0.081950 \\
& Info-graphic & $0.0766522^{*}$ & 0.0128635 & 0.000 & 0.033435 & 0.119869 \\
& Link to URL & $0.2579938^{*}$ & 0.0128635 & 0.000 & 0.214777 & 0.301211 \\
& Message & $0.2021962^{*}$ & 0.0128635 & 0.000 & 0.158979 & 0.245413 \\
& Opinion & $0.1337692^{*}$ & 0.0128635 & 0.000 & 0.090552 & 0.176986 \\
& Picture & $-0.3106768^{*}$ & 0.0128635 & 0.000 & -0.353894 & -0.267460 \\
& Picture and Text & $-0.3556346^{*}$ & 0.0128635 & 0.000 & -0.398851 & -0.312418 \\
& Podcast & $0.2533946^{*}$ & 0.0128635 & 0.000 & 0.210178 & 0.296611 \\
& Question & $0.1036928^{*}$ & 0.0128635 & 0.000 & 0.060476 & 0.146910 \\
& User Generated Content & $-0.1250286^{*}$ & 0.0128635 & 0.000 & -0.168245 & -0.081812 \\
& Video & $-0.0706916^{*}$ & 0.0128635 & 0.000 & -0.113908 & -0.027475 \\
& Mix Mode and Others & $0.2429706^{*}$ & 0.0128635 & 0.000 & 0.199754 & 0.286187 \\
& Blog Post link & 0.0253066 & 0.0128635 & 0.788 & -0.017910 & 0.068523 \\
& Call for action & $-0.2042374^{*}$ & 0.0128635 & 0.000 & -0.247454 & -0.161021 \\
& Info-graphic & $-0.1663184^{*}$ & 0.0128635 & 0.000 & -0.209535 & -0.123102 \\
& Link to URL & 0.0150232 & 0.0128635 & 0.997 & -0.028194 & 0.058240 \\
& Message & -0.0407744 & 0.0128635 & 0.088 & -0.083991 & 0.002442 \\
& Opinion & $-0.1092014^{*}$ & 0.0128635 & 0.000 & -0.152418 & -0.065985 \\
& Picture & $-0.5536474^{*}$ & 0.0128635 & 0.000 & -0.596864 & -0.510431 \\
& Picture and Text & $-0.5986052^{*}$ & 0.0128635 & 0.000 & -0.641822 & -0.555388 \\
& Podcast & 0.0104240 & 0.0128635 & 0.932 & -0.032793 & 0.053641 \\
& Question & $-0.1392778^{*}$ & 0.0128635 & 0.000 & -0.182495 & -0.096061 \\
User Generated Content & $-0.3679992^{*}$ & 0.0128635 & 0.000 & -0.411216 & -0.324782 & -0.270445 \\
& Video & $-0.3136622^{*}$ & 0.0128635 & 0.000 & -0.356879 & -0.199754 \\
\hline Video and Text & $-0.2429706^{*}$ & 0.0128635 & 0.000 & -0.286187 & \\
\hline
\end{tabular}

*. The mean difference is significant at the 0.05 level.

Table 12: No significant difference in proportional engagement for posts - FORMAT TYPE

\begin{tabular}{|c|c|}
\hline Format Type & $\begin{array}{l}\text { Statistically no significant difference in proportional } \\
\text { engagement in posts posted in the following format }\end{array}$ \\
\hline \multirow[t]{4}{*}{ Blog Post link } & Link to URL $(p=0.932)$ \\
\hline & Podcast $(\mathrm{p}=0.997)$ \\
\hline & Message $(p=0.070)$ \\
\hline & Mix Mode and Others $(\mathrm{p}=0.788)$ \\
\hline \multirow[t]{2}{*}{ Call for action } & Info-graphic $(\mathrm{p}=0.159)$ \\
\hline & Video and Text $(p=0.135)$ \\
\hline \multirow[t]{2}{*}{ Info-graphic } & Call for action $(\mathrm{p}=0.159)$ \\
\hline & Question $(p=0.700)$ \\
\hline \multirow[t]{4}{*}{ Link to URL } & Blog Post link $(p=0.932)$ \\
\hline & Podcast $(\mathrm{p}=0.932)$ \\
\hline & Message $(p=0.081)$ \\
\hline & Mix Mode and Others $(\mathrm{p}=0.997)$ \\
\hline \multirow[t]{4}{*}{ Message } & Blog Post link $(p=0.070)$ \\
\hline & Link to URL $(\mathrm{p}=0.081)$ \\
\hline & Podcast $(\mathrm{p}=0.060)$ \\
\hline & Mix Mode and Others $(\mathrm{p}=0.088)$ \\
\hline Opinion & Question $(p=0.527)$ \\
\hline Picture & Picture and Text $(\mathrm{p}=0.032)$ \\
\hline Picture and Text & Picture $(p=0.032)$ \\
\hline \multirow[t]{4}{*}{ Podcast } & Blog Post Link $(p=0.997)$ \\
\hline & Link to URL $(p=0.932)$ \\
\hline & Message $(p=0.060)$ \\
\hline & Mix Mode and Others $(\mathrm{p}=0.912)$ \\
\hline \multirow[t]{2}{*}{ Question } & Info-graphic $(p=0.700)$ \\
\hline & Opinion $(p=0.527)$ \\
\hline User Generated Content & NIL \\
\hline Video & NIL \\
\hline Video and Text & Call for action $(p=0.135)$ \\
\hline \multirow[t]{4}{*}{ Mix Mode and Others } & Blog Post link $(p=0.788)$ \\
\hline & Link to URL $(p=0.997)$ \\
\hline & Message $(p=0.088)$ \\
\hline & Podcast $(\mathrm{p}=0.932)$ \\
\hline
\end{tabular}


The Hypothesis (C) $H_{0}$ - There is no significant difference in proportional engagement for day and timing of the post was crafted to check if there was any significant difference in the proportional engagement and its relation to the weekday/weekend and the timing of the post. The descriptive statistical information given in Table 13 gives descriptive statistical information, including mean, standard deviation and $95 \%$ confidence intervals for the dependent variable- proportional engagement score- for each of the category of the Facebook post where all the 12 possibilities (weekend/weekday) with timing have been listed.

The Table 14 given the output of ANOVA analysis used to test Hypothesis (C) to understand if there is a statistically significant difference between the group means of proportional engagement scores and the day and time during which the post was displayed on Facebook. It can be inferred from the significance value 0.000 (i.e., $\mathrm{p}=0.000$ ), which is below 0.05 , that there is a statistically significant difference between in proportional engagement and the day and time of the post. This indicates that different posts displayed on Facebook at different point in time and day have different engagements. To know about those which specific posts differed in the engagement, Tukey post hoc test was used and the Table 15 gives the output which is the multiple comparisons table.

From the multiple comparisons Table 15 it can be statistically inferred that there is a statistically significant difference in proportional engagement in posts that were posted during the Week Day between 00.00 am to 08.00 am in comparison with posts displayed during any other day or time $(\mathrm{p}=0.000$ or $\mathrm{p}=0.004)$, but there is a statistically no significant difference between the posts posted on Week Day between 08.01 am to 10.00 ( $\mathrm{p}=$ 0.090 ) and also during the Week Day between $10.01 \mathrm{am}$ to $05 \# 00 \mathrm{pm}(\mathrm{p}=0.089)$.

For the posts displayed during Week Day $05.01 \mathrm{pm}$ to $08.00 \mathrm{pm}$ or during Week End between $08.01 \mathrm{am}$ to $10.00 \mathrm{am}$ OR $10.01 \mathrm{am}$ to $05 \# 00 \mathrm{pm}$ OR $05.01 \mathrm{pm}$ to $08.00 \mathrm{pm}$ it can be statistically inferred that there is a statistically significant difference in proportional engagement in posts that were posted in comparison with during the posts made at any other time $(\mathrm{p}=0.000)$. The Table 16 gives the details of the day and time during which there is a statistically no significant difference in proportional engagement in posts when compared with other day and time.

Table 13: Descriptive information - day and time of post

\begin{tabular}{|c|c|c|c|c|c|c|c|c|}
\hline \multicolumn{9}{|l|}{$\overline{\text { Engagement }}$} \\
\hline & \multirow[b]{2}{*}{$\mathrm{N}$} & \multirow[b]{2}{*}{ Mean } & \multirow[b]{2}{*}{$\begin{array}{l}\text { Std. } \\
\text { deviation }\end{array}$} & \multirow[b]{2}{*}{$\begin{array}{l}\text { Std. } \\
\text { Error }\end{array}$} & \multicolumn{2}{|c|}{$95 \%$ confidence interval for mean } & \multirow[b]{2}{*}{ Min } & \multirow[b]{2}{*}{$\operatorname{Max}$} \\
\hline & & & & & Lower bound & Upper bound & & \\
\hline Week Day $00.00 \mathrm{am}$ to $08.00 \mathrm{am}$ & 917 & 0.2488 & 0.03017 & 0.00100 & 0.2468 & 0.2507 & 0.20 & 0.30 \\
\hline Week Day 08.01 am to $10.00 \mathrm{am}$ & 1324 & 0.0492 & 0.02863 & 0.00079 & 0.0476 & 0.0507 & 0.00 & 0.10 \\
\hline Week Day 10.01 am to $05 \# 00 \mathrm{pm}$ & 6699 & 0.1050 & 0.00287 & 0.00004 & 0.1049 & 0.1051 & 0.10 & 0.11 \\
\hline Week Day $05.01 \mathrm{pm}$ to $08.00 \mathrm{pm}$ & 2312 & 0.4050 & 0.00286 & 0.00006 & 0.4049 & 0.4052 & 0.40 & 0.41 \\
\hline Week Day $10.00 \mathrm{pm}$ to $11.59 \mathrm{pm}$ & 765 & 0.8050 & 0.00291 & 0.00011 & 0.8047 & 0.8052 & 0.80 & 0.81 \\
\hline Week End 00.00 am to $08.00 \mathrm{am}$ & 342 & 0.1049 & 0.00272 & 0.00015 & 0.1046 & 0.1052 & 0.10 & 0.11 \\
\hline Week End 08.01 am to $10.00 \mathrm{am}$ & 1321 & 0.6050 & 0.00285 & 0.00008 & 0.6048 & 0.6051 & 0.60 & 0.61 \\
\hline Week End 10.01 am to $05 \# 00 \mathrm{pm}$ & 2787 & 0.5050 & 0.00284 & 0.00005 & 0.5048 & 0.5051 & 0.50 & 0.51 \\
\hline Week End $05.01 \mathrm{pm}$ to $08.00 \mathrm{pm}$ & 1089 & 0.7050 & 0.00288 & 0.00009 & 0.7049 & 0.7052 & 0.70 & 0.71 \\
\hline Week End $08.01 \mathrm{pm}$ to $10.00 \mathrm{pm}$ & 532 & 0.8489 & 0.02933 & 0.00127 & 0.8464 & 0.8514 & 0.80 & 0.90 \\
\hline Week End $10.00 \mathrm{pm}$ to $11.59 \mathrm{pm}$ & 203 & 0.8052 & 0.00284 & 0.00020 & 0.8048 & 0.8055 & 0.80 & 0.81 \\
\hline Total & 19612 & 0.3690 & 0.27196 & 0.00194 & 0.3652 & 0.3728 & 0.00 & 0.90 \\
\hline
\end{tabular}

Table 14: ANOVA analysis - DAY_TIME

\begin{tabular}{|c|c|c|c|c|c|}
\hline \multicolumn{6}{|l|}{ Engagement } \\
\hline & Sum of squares & $\mathrm{df}$ & Mean square & $\mathrm{F}$ & Sig. \\
\hline Between groups & 1447.986 & 11 & 131.635 & 1026849.020 & 0.000 \\
\hline Within groups & 2.513 & 19600 & 0.000 & & \\
\hline Total & 1450.499 & 19611 & & & \\
\hline
\end{tabular}

Table 15: Multiple comparisons of Day_Time

Dependent Variable: Engagement

Tukey HSD

\begin{tabular}{|c|c|c|c|c|c|c|}
\hline \multirow[b]{2}{*}{ (I) DAY_TIME } & \multirow[b]{2}{*}{ (J) DAY_TIME } & \multirow[b]{2}{*}{$\begin{array}{l}\text { Mean } \\
\text { difference (I-J) }\end{array}$} & \multirow[b]{2}{*}{$\begin{array}{l}\text { Std. } \\
\text { Error }\end{array}$} & \multirow[b]{2}{*}{ Sig. } & \multicolumn{2}{|c|}{ 95\% Confidence Interval } \\
\hline & & & & & Lower bound & Upper bound \\
\hline \multirow{4}{*}{$\begin{array}{l}\text { Week Day } 00.00 \mathrm{am} \\
\text { to } 08.00 \mathrm{am}\end{array}$} & Week Day 08.01 am to $10.00 \mathrm{am}$ & $0.19960^{*}$ & 0.00049 & 0.090 & 0.1980 & 0.2012 \\
\hline & Week Day 10.01 am to $05 \# 00 \mathrm{pm}$ & $0.14378^{*}$ & 0.00040 & 0.089 & 0.1425 & 0.1451 \\
\hline & Week Day $05.01 \mathrm{pm}$ to $08.00 \mathrm{pm}$ & $-0.15626^{*}$ & 0.00044 & 0.000 & -0.1577 & -0.1548 \\
\hline & Week Day $08.01 \mathrm{pm}$ to $10.00 \mathrm{pm}$ & $-0.55617^{*}$ & 0.00049 & 0.000 & -0.5578 & -0.5546 \\
\hline
\end{tabular}


Table 15: Continue

Week Day 08.01 am to $10.00 \mathrm{am}$

Week Day 10.01 am to $05 \# 00 \mathrm{pm}$

Week Day $05.01 \mathrm{pm}$ to $08.00 \mathrm{pm}$

Week Day $08.01 \mathrm{pm}$ to $10.00 \mathrm{pm}$

Week Day $10.00 \mathrm{pm}$ to $11.59 \mathrm{pm}$

Week End 00.00 am to $08.00 \mathrm{am}$
Week Day $10.00 \mathrm{pm}$ to $11.59 \mathrm{pm}$

Week End $00.00 \mathrm{am}$ to $08.00 \mathrm{am}$

Week End 08.01 am to $10.00 \mathrm{am}$

Week End 10.01 am to $05 \# 00 \mathrm{pm}$

Week End $05.01 \mathrm{pm}$ to $08.00 \mathrm{pm}$

Week End $08.01 \mathrm{pm}$ to $10.00 \mathrm{pm}$

Week End $10.00 \mathrm{pm}$ to $11.59 \mathrm{pm}$

Week Day 00.00 am to $08.00 \mathrm{am}$

Week Day 10.01 am to $05 \# 00 \mathrm{pm}$

Week Day $05.01 \mathrm{pm}$ to $08.00 \mathrm{pm}$

Week Day $08.01 \mathrm{pm}$ to $10.00 \mathrm{pm}$

Week Day $10.00 \mathrm{pm}$ to $11.59 \mathrm{pm}$

Week End 00.00 am to $08.00 \mathrm{am}$

Week End 08.01 am to 10.00 am

Week End 10.01 am to $05 \# 00$ pm

Week End $05.01 \mathrm{pm}$ to $08.00 \mathrm{pm}$

Week End $08.01 \mathrm{pm}$ to $10.00 \mathrm{pm}$

Week End $10.00 \mathrm{pm}$ to $11.59 \mathrm{pm}$

Week Day 00.00 am to $08.00 \mathrm{am}$

Week Day 08.01 am to $10.00 \mathrm{am}$

Week Day $05.01 \mathrm{pm}$ to $08.00 \mathrm{pm}$

Week Day 08.01 pm to $10.00 \mathrm{pm}$

Week Day $10.00 \mathrm{pm}$ to $11.59 \mathrm{pm}$

Week End $00.00 \mathrm{am}$ to $08.00 \mathrm{am}$

Week End 08.01 am to $10.00 \mathrm{am}$

Week End 10.01 am to $05 \# 00 \mathrm{pm}$

Week End $05.01 \mathrm{pm}$ to $08.00 \mathrm{pm}$

Week End $08.01 \mathrm{pm}$ to $10.00 \mathrm{pm}$

Week End $10.00 \mathrm{pm}$ to $11.59 \mathrm{pm}$

Week Day 00.00 am to $08.00 \mathrm{am}$

Week Day 08.01 am to $10.00 \mathrm{am}$

Week Day 10.01 am to $05 \# 00 \mathrm{pm}$

Week Day $08.01 \mathrm{pm}$ to $10.00 \mathrm{pm}$

Week Day $10.00 \mathrm{pm}$ to $11.59 \mathrm{pm}$

Week End 00.00 am to $08.00 \mathrm{am}$

Week End 08.01 am to $10.00 \mathrm{am}$

Week End 10.01 am to $05 \# 00 \mathrm{pm}$

Week End $05.01 \mathrm{pm}$ to $08.00 \mathrm{pm}$

Week End $08.01 \mathrm{pm}$ to $10.00 \mathrm{pm}$

Week End 10.00 pm to $11.59 \mathrm{pm}$

Week Day 00.00 am to $08.00 \mathrm{am}$

Week Day $08.01 \mathrm{am}$ to $10.00 \mathrm{am}$

Week Day 10.01 am to $05 \# 00 \mathrm{pm}$

Week Day $05.01 \mathrm{pm}$ to $08.00 \mathrm{pm}$

Week Day $10.00 \mathrm{pm}$ to $11.59 \mathrm{pm}$

Week End 00.00 am to $08.00 \mathrm{am}$

Week End 08.01 am to $10.00 \mathrm{am}$

Week End 10.01 am to $05 \# 00 \mathrm{pm}$

Week End $05.01 \mathrm{pm}$ to $08.00 \mathrm{pm}$

Week End $08.01 \mathrm{pm}$ to $10.00 \mathrm{pm}$

Week End $10.00 \mathrm{pm}$ to $11.59 \mathrm{pm}$

Week Day 00.00 am to $08.00 \mathrm{am}$

Week Day 08.01 am to $10.00 \mathrm{am}$

Week Day 10.01 am to $05 \# 00 \mathrm{pm}$

Week Day $05.01 \mathrm{pm}$ to $08.00 \mathrm{pm}$

Week Day $08.01 \mathrm{pm}$ to $10.00 \mathrm{pm}$

Week End 00.00 am to $08.00 \mathrm{am}$

Week End 08.01 am to $10.00 \mathrm{am}$

Week End 10.01 am to $05 \# 00 \mathrm{pm}$

Week End $05.01 \mathrm{pm}$ to $08.00 \mathrm{pm}$

Week End $08.01 \mathrm{pm}$ to $10.00 \mathrm{pm}$

Week End $10.00 \mathrm{pm}$ to $11.59 \mathrm{pm}$

Week Day 00.00 am to $08.00 \mathrm{am}$

Week Day $08.01 \mathrm{am}$ to $10.00 \mathrm{am}$

Week Day 10.01 am to $05 \# 00 \mathrm{pm}$

\begin{tabular}{|c|c|}
\hline$-0.55616^{*}$ & 0.00055 \\
\hline $0.14391^{*}$ & 0.00072 \\
\hline$-0.35618^{*}$ & 0.00049 \\
\hline$-0.25617^{*}$ & 0.00043 \\
\hline$-0.45624^{*}$ & 0.00051 \\
\hline$-0.60007^{*}$ & 0.00062 \\
\hline$-0.55637^{*}$ & 0.00088 \\
\hline$-0.19960^{*}$ & 0.00049 \\
\hline$-0.05583^{*}$ & 0.00034 \\
\hline$-0.35586^{*}$ & 0.00039 \\
\hline$-0.75577^{*}$ & 0.00044 \\
\hline$-0.75577^{*}$ & 0.00051 \\
\hline$-0.05569^{*}$ & 0.00069 \\
\hline$-0.55578^{*}$ & 0.00044 \\
\hline$-0.45577^{*}$ & 0.00038 \\
\hline$-0.65584^{*}$ & 0.00046 \\
\hline$-0.79967^{*}$ & 0.00058 \\
\hline$-0.75597^{*}$ & 0.00085 \\
\hline$-0.14378^{*}$ & 0.00040 \\
\hline $0.05583^{*}$ & 0.00034 \\
\hline$-0.30003^{*}$ & 0.00027 \\
\hline$-0.69994^{*}$ & 0.00034 \\
\hline$-0.69994^{*}$ & 0.00043 \\
\hline 0.00014 & 0.00063 \\
\hline$-0.49996^{*}$ & 0.00034 \\
\hline$-0.39994^{*}$ & 0.00026 \\
\hline$-0.60001^{*}$ & 0.00037 \\
\hline$-0.74384^{*}$ & 0.00051 \\
\hline$-0.70014^{*}$ & 0.00081 \\
\hline $0.15626^{*}$ & 0.00044 \\
\hline $0.35586^{*}$ & 0.00039 \\
\hline $0.30003^{*}$ & 0.00027 \\
\hline$-0.39991^{*}$ & 0.00039 \\
\hline$-0.39990^{*}$ & 0.00047 \\
\hline $0.30017^{*}$ & 0.00066 \\
\hline$-0.19992^{*}$ & 0.00039 \\
\hline$-0.09991^{*}$ & 0.00032 \\
\hline$-0.29998^{*}$ & 0.00042 \\
\hline$-0.44381^{*}$ & 0.00054 \\
\hline$-0.40011^{*}$ & 0.00083 \\
\hline $0.55617^{*}$ & 0.00049 \\
\hline $0.75577^{*}$ & 0.00044 \\
\hline $0.69994^{*}$ & 0.00034 \\
\hline $0.39991^{*}$ & 0.00039 \\
\hline 0.00000 & 0.00051 \\
\hline $0.70008^{*}$ & 0.00069 \\
\hline $0.19999^{*}$ & 0.00044 \\
\hline $0.30000^{*}$ & 0.00038 \\
\hline $0.09993^{*}$ & 0.00046 \\
\hline$-0.04390^{*}$ & 0.00058 \\
\hline-0.00020 & 0.00085 \\
\hline $0.55616^{*}$ & 0.00055 \\
\hline $0.75577^{*}$ & 0.00051 \\
\hline $0.69994^{*}$ & 0.00043 \\
\hline $0.39990^{*}$ & 0.00047 \\
\hline 0.00000 & 0.00051 \\
\hline $0.70007^{*}$ & 0.00074 \\
\hline $0.19998^{*}$ & 0.00051 \\
\hline $0.30000^{*}$ & 0.00046 \\
\hline $0.09992^{*}$ & 0.00053 \\
\hline$-0.04390^{*}$ & 0.00064 \\
\hline-0.00020 & 0.00089 \\
\hline$-0.14391^{*}$ & 0.00072 \\
\hline $0.05569^{*}$ & 0.00069 \\
\hline-0.00014 & 0.00063 \\
\hline
\end{tabular}

0.000

0.000

0.000

0.000

0.000

0.000

0.004

0.090

0.083

0.000

0.000

0.000

0.000

0.000

0.000

0.000

0.000

0.000

0.000

0.083

0.000

0.000

0.000

0.089

0.000

0.000

0.000

0.000

0.000

0.000

0.000

0.000

0.000

0.000

0.000

0.000

0.000

0.000

0.000

0.000

0.000

0.000

0.000

0.000

0.976

0.000

0.000

0.000

0.000

0.000

0.976

0.000

0.000

0.000

0.000

0.976

0.000

0.000

0.000

0.000

0.000

0.976

0.000

0.000

0.976

$\begin{array}{ll}-0.5580 & -0.5544 \\ 0.1416 & 0.1463 \\ -0.3578 & -0.3546 \\ -0.2576 & -0.2548 \\ -0.4579 & -0.4546 \\ -0.6021 & -0.598 \\ -0.5592 & -0.5535 \\ -0.2012 & -0.198 \\ -0.0569 & -0.0547 \\ -0.3571 & -0.3546 \\ -0.7572 & -0.7543 \\ -0.7574 & -0.7541 \\ -0.0579 & -0.0534 \\ -0.5572 & -0.5543\end{array}$

$-0.5572$

$-0.4570 \quad-0.4545$

$-0.6574-0.6543$

$-0.8016 \quad-0.7978$

$-0.7588 \quad-0.7532$

$-0.1451 \quad-0.1425$

$0.0547 \quad 0.0569$

$-0.3009 \quad-0.2991$

$-0.7011 \quad-0.6988$

$-0.7014 \quad-0.6985$

$-0.0019 \quad 0.0022$

$-0.5011 \quad-0.4988$

$-0.4008 \quad-0.3991$

$-0.6012 \quad-0.5988$

$-0.7455 \quad-0.7422$

$\begin{array}{ll}-0.7028 & -0.6975\end{array}$

$0.1548 \quad 0.1577$

$0.3546 \quad 0.3571$

$0.2991 \quad 0.3009$

$-0.4012 \quad-0.3986$

$-0.4014 \quad-0.3984$

$0.2980 \quad 0.3023$

$-0.2012 \quad-0.1986$

$-0.1009 \quad-0.0989$

$-0.3013 \quad-0.2986$

$-0.4456 \quad-0.4420$

$-0.4028 \quad-0.3974$

$0.5546 \quad 0.5578$

$0.7543 \quad 0.7572$

$0.6988 \quad 0.7011$

$\begin{array}{ll}0.3986 & 0.4012\end{array}$

$\begin{array}{ll}-0.0017 & 0.0017\end{array}$

$0.6978 \quad 0.7023$

$0.1985 \quad 0.2014$

$\begin{array}{ll}0.2988 & 0.3012\end{array}$

$0.0984 \quad 0.1014$

$-0.0458 \quad-0.0420$

$-0.0030 \quad 0.0026$

$\begin{array}{ll}-0.5544 & 0.5580\end{array}$

$0.7541 \quad 0.7574$

$0.6985 \quad 0.7014$

$0.3984 \quad 0.4014$

$\begin{array}{ll}-0.0017 & 0.0017\end{array}$

$0.6977 \quad 0.7025$

$0.1983 \quad 0.2017$

$0.2985 \quad 0.3015$

$0.0982 \quad 0.1017$

$-0.0460 \quad-0.0418$

$-0.0031 \quad 0.0027$

$-0.1463 \quad-0.1416$

$0.0534 \quad 0.0579$

$-0.0022$

0.0019 
Table 15: Continue

Week End 08.01 am to $10.00 \mathrm{am}$

Week End 10.01 am to $05 \# 00 \mathrm{pm}$

Week End $05.01 \mathrm{pm}$ to $08.00 \mathrm{pm}$

Week End $08.01 \mathrm{pm}$ to $10.00 \mathrm{pm}$

Week End 10.00 pm to $11.59 \mathrm{pm}$
Week Day 05.01 pm to $08.00 \mathrm{pm}$

Week Day 08.01 pm to $10.00 \mathrm{pm}$

Week Day $10.00 \mathrm{pm}$ to $11.59 \mathrm{pm}$

Week End 08.01 am to $10.00 \mathrm{am}$

Week End 10.01 am to $05 \# 00 \mathrm{pm}$

Week End $05.01 \mathrm{pm}$ to $08.00 \mathrm{pm}$

Week End $08.01 \mathrm{pm}$ to $10.00 \mathrm{pm}$

Week End 10.00 pm to $11.59 \mathrm{pm}$

Week Day 00.00 am to $08.00 \mathrm{am}$

Week Day 08.01 am to $10.00 \mathrm{am}$

Week Day 10.01 am to $05 \# 00 \mathrm{pm}$

Week Day $05.01 \mathrm{pm}$ to $08.00 \mathrm{pm}$

Week Day $08.01 \mathrm{pm}$ to $10.00 \mathrm{pm}$

Week Day $10.00 \mathrm{pm}$ to $11.59 \mathrm{pm}$

Week End 00.00 am to $08.00 \mathrm{am}$

Week End 10.01 am to $05 \# 00 \mathrm{pm}$

Week End $05.01 \mathrm{pm}$ to $08.00 \mathrm{pm}$

Week End $08.01 \mathrm{pm}$ to $10.00 \mathrm{pm}$

Week End 10.00 pm to $11.59 \mathrm{pm}$

Week Day 00.00 am to $08.00 \mathrm{am}$

Week Day 08.01 am to $10.00 \mathrm{am}$

Week Day 10.01 am to $05 \# 00 \mathrm{pm}$

Week Day $05.01 \mathrm{pm}$ to $08.00 \mathrm{pm}$

Week Day $08.01 \mathrm{pm}$ to $10.00 \mathrm{pm}$

Week Day $10.00 \mathrm{pm}$ to $11.59 \mathrm{pm}$

Week End 00.00 am to 08.00 am

Week End 08.01 am to $10.00 \mathrm{am}$

Week End $05.01 \mathrm{pm}$ to $08.00 \mathrm{pm}$

Week End 08.01 pm to $10.00 \mathrm{pm}$

Week End $10.00 \mathrm{pm}$ to $11.59 \mathrm{pm}$

Week Day 00.00 am to $08.00 \mathrm{am}$

Week Day 08.01 am to $10.00 \mathrm{am}$

Week Day 10.01 am to $05 \# 00$ pm

Week Day $05.01 \mathrm{pm}$ to $08.00 \mathrm{pm}$

Week Day $08.01 \mathrm{pm}$ to $10.00 \mathrm{pm}$

Week Day $10.00 \mathrm{pm}$ to $11.59 \mathrm{pm}$

Week End $00.00 \mathrm{am}$ to $08.00 \mathrm{am}$

Week End 08.01 am to $10.00 \mathrm{am}$

Week End 10.01 am to $05 \# 00 \mathrm{pm}$

Week End $08.01 \mathrm{pm}$ to $10.00 \mathrm{pm}$

Week End $10.00 \mathrm{pm}$ to $11.59 \mathrm{pm}$

Week Day 00.00 am to $08.00 \mathrm{am}$

Week Day 08.01 am to $10.00 \mathrm{am}$

Week Day 10.01 am to $05 \# 00 \mathrm{pm}$

Week Day 05.01 pm to $08.00 \mathrm{pm}$

Week Day $08.01 \mathrm{pm}$ to $10.00 \mathrm{pm}$

Week Day $10.00 \mathrm{pm}$ to $11.59 \mathrm{pm}$

Week End 00.00 am to $08.00 \mathrm{am}$

Week End 08.01 am to $10.00 \mathrm{am}$

Week End 10.01 am to $05 \# 00 \mathrm{pm}$

Week End $05.01 \mathrm{pm}$ to $08.00 \mathrm{pm}$

Week End $10.00 \mathrm{pm}$ to $11.59 \mathrm{pm}$

Week Day 00.00 am to $08.00 \mathrm{am}$

Week Day 08.01 am to $10.00 \mathrm{am}$

Week Day 10.01 am to $05 \# 00 \mathrm{pm}$

Week Day $05.01 \mathrm{pm}$ to $08.00 \mathrm{pm}$

Week Day 08.01 pm to $10.00 \mathrm{pm}$

Week Day $10.00 \mathrm{pm}$ to $11.59 \mathrm{pm}$

Week End 00.00 am to 08.00 am

Week End 08.01 am to $10.00 \mathrm{am}$

Week End 10.01 am to $05 \# 00$ pm

Week End $05.01 \mathrm{pm}$ to $08.00 \mathrm{pm}$

Week End $08.01 \mathrm{pm}$ to $10.00 \mathrm{pm}$
$-0.30017^{*}$

$-0.70008^{*}$

$-0.70007$

$-0.50009^{*}$

$-0.40008^{*}$

$-0.60015^{*}$

$-0.74398^{*}$

$-0.70028^{*}$

$0.35618^{*}$

$0.55578^{*}$

$0.49996^{*}$

$0.19992^{*}$

$-0.19999^{*}$

$-0.19998^{*}$

$0.50009^{*}$

$0.10001^{*}$

$-0.10006^{*}$

$-0.24389^{*}$

$-0.20019^{*}$

$0.25617^{*}$

$0.45577^{*}$

$0.39994^{*}$

$0.09991^{*}$

$-0.30000$

$-0.30000$

$0.40008^{*}$

$-0.10001^{*}$

$-0.20007^{*}$

$-0.34390$

$-0.30020^{*}$

$0.45624^{*}$

$0.65584^{*}$

$0.60001^{*}$

$0.29998^{*}$

$-0.09993$

$-0.09992$

$0.60015^{*}$

$0.10006^{*}$

$0.20007^{*}$

$-0.14383^{*}$

$-0.10013^{*}$

$0.60007^{*}$

$0.79967^{*}$

$0.74384^{*}$

$0.44381^{*}$

$0.04390^{*}$

$0.04390^{*}$

$0.74398^{*}$

$0.24389^{*}$

$0.34390^{*}$

$0.14383^{*}$

$0.04370^{*}$

$0.55637^{*}$

$0.75597^{*}$

$0.70014^{*}$

$0.40011^{*}$

0.00020

0.00020

$0.70028^{*}$

$0.20019^{*}$

$0.30020^{*}$

$0.10013^{*}$

$-0.04370^{*}$

\subsection{6}

0.00069

0.00074

0.00069

0.00065

0.00070

0.00078

0.00100

0.00049

0.00044

0.00034

0.00039

0.00044

0.00051

0.00069

0.00038

0.00046

0.00058

0.00085

0.00043

0.00038

0.00026

0.00032

0.00038

0.00046

0.00065

0.00038

0.00040

0.00054

0.00082

0.00051

0.00046

0.00037

0.00042

0.00046

0.00053

0.00070

0.00046

0.00040

0.00060

0.00087

0.00062

0.00058

0.00051

0.00054

0.00058

0.00064

0.00078

0.00058

0.00054

0.00060

0.00093

0.00088

0.00085

0.00081

0.00083

0.00085

0.00089

0.00100

0.00085

0.00082

0.00087

0.00093

\subsection{0}

0.000

0.000

0.000

0.000

0.000

0.000

0.000

0.000

0.000

0.000

0.000

0.000

0.000

0.000

0.000

0.000

0.000

0.000

0.000

0.000

0.000

0.000

0.000

0.000

0.000

0.000

0.000

0.000

0.000

0.000

0.000

0.000

0.000

0.000

0.000

0.000

0.000

0.000

0.000

0.000

0.000

0.000

0.000

0.000

0.000

0.000

0.000

0.000

0.000

0.000

0.000

0.000

0.000

0.000

0.000

0.976

0.976

0.000

0.000

0.000

0.000

0.000

$-0.3023$

$-0.7023$

$-0.7025$

$-0.5023$

$-0.4022$

$-0.6024$

$-0.7465$

$-0.7036$

0.3546

0.5543

0.4988

0.1986

$-0.2014$

$-0.2017$

0.4978

0.0988

$-0.1016$

$-0.2458$

$-0.2030$

0.2548

0.4545 
Table 16: No significant difference in proportional engagement for posts - DAY_TIME

\begin{tabular}{|c|c|}
\hline Format type & $\begin{array}{l}\text { Statistically NO significant difference in proportional } \\
\text { engagement in posts posted during the following day and time }\end{array}$ \\
\hline Week Day 00.00 am to $08.00 \mathrm{am}$ & $\begin{array}{l}\text { Week Day } 10.01 \text { am to } 05 \# 00 \mathrm{pm}(\mathrm{p}=0.089) \\
\text { Week Day } 08.01 \text { am to } 10.00 \mathrm{am}(\mathrm{p}=0.090)\end{array}$ \\
\hline Week Day 08.01 am to 10.00 am & $\begin{array}{l}\text { Week Day } 00.00 \text { am to } 08.00 \text { am }(\mathrm{p}=0.090) \\
\text { Week Day } 10.01 \text { am to } 05 \# 00 \mathrm{pm}(\mathrm{p}=0.083)\end{array}$ \\
\hline Week Day 10.01 am to $05 \# 00 \mathrm{pm}$ & $\begin{array}{l}\text { Week Day } 08.01 \text { am to } 10.00 \mathrm{am}(\mathrm{p}=0.083) \\
\text { Week End } 00.00 \text { am to } 08.00 \mathrm{am}(\mathrm{p}=0.089)\end{array}$ \\
\hline Week Day $05.01 \mathrm{pm}$ to $08.00 \mathrm{pm}$ & NIL \\
\hline Week Day $08.01 \mathrm{pm}$ to $10.00 \mathrm{pm}$ & $\begin{array}{l}\text { Week Day } 10.00 \mathrm{pm} \text { to } 11.59 \mathrm{pm}(\mathrm{p}=0.976) \\
\text { Week End } 10.00 \mathrm{pm} \text { to } 11.59 \mathrm{pm}(\mathrm{p}=0.976)\end{array}$ \\
\hline Week Day $10.00 \mathrm{pm}$ to $11.59 \mathrm{pm}$ & $\begin{array}{l}\text { Week Day } 08.01 \mathrm{pm} \text { to } 10.00 \mathrm{pm}(\mathrm{p}=0.976) \\
\text { Week End } 10.00 \mathrm{pm} \text { to } 11.59 \mathrm{pm}(\mathrm{p}=0.976)\end{array}$ \\
\hline Week End 00.00 am to 08.00 am & Week Day 10.01 am to $05 \# 00 \mathrm{pm}(\mathrm{p}=0.976)$ \\
\hline Week End 08.01 am to $10.00 \mathrm{am}$ & NIL \\
\hline Week End 10.01 am to $05 \# 00 \mathrm{pm}$ & NIL \\
\hline Week End $05.01 \mathrm{pm}$ to $08.00 \mathrm{pm}$ & NIL \\
\hline Week End $10.00 \mathrm{pm}$ to $11.59 \mathrm{pm}$ & $\begin{array}{l}\text { Week Day } 08.01 \mathrm{pm} \text { to } 10.00 \mathrm{pm}(\mathrm{p}=0.976) \\
\text { Week Day } 10.00 \mathrm{pm} \text { to } 11.59 \mathrm{pm}(\mathrm{p}=0.976)\end{array}$ \\
\hline
\end{tabular}

\section{Results and Discussion}

The analysis gives a clear indication to arrive at strategies which can help B school to ensure higher engagement for the posts displayed on Facebook. Some strategies that could be followed are listed below which can be of immense use.

Strategy 1 - Selectively Choose and Promote Specific Posts on Facebook for Better Engagements

From the analysis of data it can be concluded that B school can choose and post content related to Alumni, Students academic achievement, B school ranking, Corporate Case Competition related information and posts related to startup information to ensure that the engagement is higher (Table 8). If this strategy is adopted then, the posts will have significantly higher engagements when compared to other type of posts.

\section{Strategy 2 - Choose the Format of the Post as Video or User Generated Content}

The analysis have revealed that the posts that have Video content or those which are user generated have significantly higher engagement (Table 12). If the information in the form of videos is created and posted, it would attract higher engagement. If the video are posted by students or those who are members on the Facebook page then it would be attracting higher engagement as it would then become user generated content.

Strategy 3 - Choose the Appropriate Day and Time for the Post to Ensure Higher Engagements

The analysis clearly indicates that posts made during weekday between $05.01 \mathrm{pm}$ to $08.00 \mathrm{pm}$ and also during weekends between 08.01 am to 10.00 am OR between 10.01 am to $05 \# 00 \mathrm{pm}$ OR between $05.01 \mathrm{pm}$ to 08.00 pm attract higher engagements (Table 16). This clearly indicates that posts displayed during weekends can attract higher engagements.

\section{Strategy 4 - Mix the Strategies and Plan the Posting to Ensure Higher Engagements}

To ensure better reach and engagement of the posts, the strategies (1,2 and 3) listed above can be mixed and adopted. A video post which is on alumni posted on a week end between $05.01 \mathrm{pm}$ to $08.00 \mathrm{pm}$ would be a classic example of mixed mode strategy.

\section{Conclusion}

This paper has important contributions to B schools and higher educational institutions who spend significant amount of money in digital marketing. It also adds value to the existing body of knowledge related to the social media space and its use in higher education marketing. More importantly the paper adds significant value to the existing literature on how content and format of social media a can influence engagement of users. This could be used by digital marketing managers for their practical applications.

Strategies have been suggested on the nature and format of content and day and time of the post which can help in increasing engagement of post on Facebook, which can help in significantly increasing the return on investment in the digital spends of marketing. By implementing the strategies suggested the visibility of the posts can be increased and also lead to higher engagement of users on Facebook.

\section{Limitation and Scope of Further Work}

This research paper does not take user behavior and user experience into consideration. Further 
research can be taken to understand the user experience using experiments. As all social media networks including Facebook are frequently tweaking their algorithms, this study might not be valid for all social media networks. Also this study was based on India specific B schools and their official Facebook page content. The variables defined for the study are India specific. Further studies can be taken to check if the variables change based on the country in which the higher educational institutions or B Schools operate. Also a comparative study of B Schools from different countries can also be taken up.

\section{Acknowledgment}

The authors would like to thank National Instituton Ranking Framework (NIRF) Government of India for publsihing the B School Ranking which made it possible for this research work to be taken up and it was easy to select the B School for the research.

\section{Author's Contributions}

Vikram Kumar: The prime author for the research work.

Dr Ramakrishnana Raman: Provided guidance for the research work and supported in statistical analysis.

\section{Ethics}

This article is original and contains unpublished material. The authors confirm to have read and approved the manuscript and there are no ethical issues involved.

\section{References}

Anderson, G., 2008. Mapping academic resistance in the managerial university. Organization, 15: 251-270.

Apoorva, V.K., J. Shaji, R. Raman, B.S. Vijayakumar and G. Anuradha et al., 2013. Blog content and user engagement - an insight using statistical analysis. Int. J. Eng. Technol., 5: 2719-2733

Arun, T. and M.J. Xavier, 2013. Determinants of customers' online purchase intention: An empirical study in India. J. Indian Bus. Res., 5: 17-32.

Augustsson, G., 2010. Web 2.0, pedagogical support for reflexive and emotional social interaction among Swedish students. Internet Higher Educ., 13: 197-205.

Barker, V., 2009. Older adolescents' motivations for social network site use: The influence of gender, group identity and collective self-esteem. Cyber Psychol. Behav., 12: 209-213.

Barnes, N.G. and E. Mattson, 2009a. Social media and college admissions: The first longitudinal study. University of Massachusetts, Center for Marketing Research, Dartmouth, NH.
Barnes, N.G. and E. Mattson, 2009b. Social media in the 2009 Inc. 500: New tools and new trends. Center for Marketing Research, University of Massachusetts Dartmouth.

Barnes, N.G. and E. Mattson, 2010. Social media and college admissions: Higher-ed beats business in adoption of new tools for third year. University of Massachusetts, Center for Marketing Research, Dartmouth, NH.

Boyd, D., 2008. Why youth (heart) social network sites: The role of Networked Publics. In: Youth, Identity and Digital Media, Buckingham, D. (Ed.), The MIT Press, Cambridge, pp: 119-142.

Constantinides, E. and S.J. Fountain, 2008. Web 2.0: Conceptual foundations and marketing issues. J. Direct Data Digital Market. Pract., 9: 231-244.

Constantinides, E., 2010. Connecting Small and Medium Enterprises to the New Consumer: The Web 2.0 as Marketing Tool. In: Global Perspectives on Small and Medium Enterprise, Bharati, P., I. Lee and A. Chaudhury (Eds.), IGI Global, Hershey, Pennsylvania.

De Clerck, J., 2013. This is your real social business strategy challenge. I-Scoop.

Dunay, P., 2014. From employee to advocate: Mobilize your team to share your brand content. Social Media Today.

Edmiston-Strasser, D.M., 2009. An examination of integrated marketing communication in U.S. public institutions of higher education. J. Market. Higher Educ., 19: 142-165.

Eikelmann, S., J. Hajj and M. Peterson, 2008. Opinion piece: Web 2.0: Profiting from the threat. J. Direct Data Digital Market. Pract., 9: 293-295.

Ghauri, P., C. Lutz and G. Tesfom, 2003. Using networks to solve export-marketing problems of small-and medium-sized firms from developing countries. Eur. J. Market., 37: 728-752.

Gibbs, P. and P. Murphy, 2009. Implementation of ethical higher education marketing. Tertiary Educ. Manage., 15: 341-354.

Gibbs, P., 2002. From the invisible hand to the invisible handshake: Marketing higher education. Res. PostCompulsory Educ., 7: 325-338.

Gronroos, C., 1994. Quo vadis, marketing? Toward a relationship marketing paradigm. J. Market. Manage., 10: 347-360.

Harsha, P.P. and S. Shah, 2011. Creating brand value of higher education institution. IJMT, 19: 152-158.

Hayes, T.J., D. Ruschman and M.M. Walker, 2009. Social networking as an admission tool: A case study in success. J. Market. Higher Educ., 19: 109-124.

Helgesen, Ø., 2008. Marketing for higher education: A relationship marketing approach. J. Market. Higher Educ., 18: 50-78. 
Hemsley-Brown, J.V. and I. Oplatka, 2006. Universities in a competitive global marketplace: A systematic review of the literature on higher education marketing. Int. J. Public Sector Manage., 19: 316-338.

Jongbloed, B., 2003. Marketisation in higher education, Clark's triangle and the essential ingredients of markets. Higher Educ. Q., 57: 110-135

Joshi, A. 2018. Vedantic applications of augmented reality for strategic social marketing campaigns in India. J. Indian Bus. Res., 10: 256-273.

Kabilan, M.K., N. Ahmad and M.J.Z. Abidin, 2010. Facebook: An online environment for learning of English in institutions of higher education? Internet Higher Educ., 13: 179-187.

Kaplan, A.M. and M. Haenlein, 2010. Users of the world, unite! The challenges and opportunities of social media. Bus. Horizons, 53: 59-68.

Karin, I. and R. Eiferman, 2006. Technology-Based Marketing (TBM): A new competitive approach for high-tech industries. Int. J. Global Bus. Competitiveness, 2: 19-25.

Kim, W., O.R. Jeong and S.W. Lee, 2010. On social web sites. Inform. Syst., 35: 215-236.

Klassen, M.L., 2002. Relationship marketing on the Internet: The case of top- and lower-ranked US universities and colleges. J. Retail. Consumer Services, 9: 81-85.

Kolbitsch, J. and H. Maurer, 2006. The transformation of the web: How emerging communities shape the information we consume. J. Univ. Comput. Sci., 12: 187-213.

Kumar, S. and M.K. Dash, 2011. Management education in India: Trends, issues and implications. Res. J. Int. Stud., 18: 15-25.

Kwok, L. and B. Yu, 2013. Spreading social media messages on Facebook: An analysis of restaurant business-to-consumer communications. Cornell Hospitality Q, 54: 84-94.

Li, H., S.M. Edwards and J.H. Lee, 2002. Measuring the intrusiveness of advertisements: Scale development and validation. J. Advert., 31: 37-47.

Lindbeck, R. and B. Fodrey, 2010. Using technology in undergraduate admission: A student perspective. J. Coll. Admiss., 208: 10-17.

Madhavan, P., L. Vicki and L.S. Mary, 2015. A timebased analysis of changing consumer values in India. J. Indian Bus. Res., 7: 271-291.

Mangold, W.G. and D.J. Faulds, 2009. Social media: The new hybrid element of the promotion mix. Bus. Horizons, 52: 357-365.

Maringe, F., 2006. University and course choice: Implications for positioning, recruitment and marketing. Int. J. Educ. Manage., 20: 466-479.

McAlexander, J.H. and H.F. Koenig, 2001. University experiences, the student-college relationship and alumni support. J. Market. Higher Educ., 10: 21-43.
McCoy, S., A. Everard, P. Polak and D.F. Galletta, 2007. The effects of online advertising. Commun. ACM, 50: 84-88.

Nambisan, S. and P. Nambisan, 2008. How to profit from a better virtual customer environment. MIT Sloan Manage. Rev., 49: 53-53.

Nevzat, R., Y. Amca, C. Tanova and H. Amca, 2016. Role of social media community in strengthening trust and loyalty for a university. Comput. Human Behav., 65: 550-559.

Peppers, D. and M. Rogers, 1993. A new marketing paradigm: Share of customer, not market share. Strategy Leadership, 23: 14-18.

Peruta, A. and A.B. Shields, 2016. Social media in higher education: Understanding how colleges and universities use Facebook. J. Market. Higher Educ., 27: 1-13.

Peruta, A. and C. Helm, 2018a. Engaging university alumni through social media: Strategies for creating community. J. Soc. Media Society, 7: 123-150.

Peruta, A. and C. Helm, 2018b. University Facebook pages: Engaging the alumni community in the digital era. J. Soc. Media Society, 7: 123-150.

Philip, J., 2008. Management education in India. Proceedings of the 13th International Study and Practical Conference - Competitiveness in Information Society: BRICS-countries Experience, (ISE' 08), State University of Management, Moscow, Russia.

Rakhi, T. and S. Mala, 2013. Customer usage intention of mobile commerce in India: An empirical study. J. Indian Bus. Res., 5: 52-72.

Ranchhod, A., 2007. Developing mobile marketing strategies. Int. J. Mobile Advertis., 2: 76-83.

Sandvig, J.C., 2016. The role of social media in college recruiting. Int. J. Web Based Communities, 12: 23-34.

Schultz, D.E. and H.F. Schultz, 2004. IMC: The next generation. McGraw-Hill, New York.

Shah, A., 2012. India's business schools get tough lesson in supply and demand. Reuters.

Sinha, D.P., 2006. Management Education in India: Perspectives and Challenges. 1st Edn., ICFAI University Press, Hyderabad, India, ISBN-13: 9788178812793.

Spaulding, T.J., 2010. How can virtual communities create value for business? Electronic Commerce Res. Applic., 9: 38-49.

Stryker, S., 1968. Identity salience and role performance: The relevance of symbolic interaction theory for family research. J. Marriage Family, 30: 558-564

Thomas, A.R., 2007. The end of mass marketing: Or, why all successful marketing is now direct marketing. Direct Marketing: Int. J., 1: 6-16.

Weiss, M., 2008. Results-based interaction design. Educ. $\mathrm{Q}, 31: 42-49$

William, J., 1950. The principles of psychology. New York, Dover Publications. 


\section{Electronic Source}

Adage, 2018. http://www.adageindia.in/digital/brandsorganic-facebook-reach-has-crashed-since-octoberstudy/articleshow/45747351.cms

Bazaarvoice, www.bazaarvoice.com/static/download/Bazaarvoice _Millennials_Infographic.pdf

Brandwatch, 2019. https://www.brandwatch.com/blog/facebookstatistics/

Buddy

Media,

2011

http://www.buddymedia.com/newsroom/2011/09/bu ddymedia-releases-new-research-paper-a-statisticalreview-for-the-retail-industry-strategiesforeffective-facebook-wall-posts/

Lee, A., 2012. 8 surefire ways to increase engagement on Facebook.

Kissmetrics. https://blog.kissmetrics.com/increase-engagementon-facebook/
NIRF

Ranking, 2018 https://www.nirfindia.org/2018/ManagementRankin g.html

Pew Research Center https://www.pewresearch.org/facttank/2019/03/07/7-key-findings-about-mobilephone-and-social-media-use-in-emergingeconomies/

Statista

Research. https://www.statista.com/statistics/268136/top-15countries-based-on-number-of-facebook-users/

Vahl, A., 2015. 11 Ways to boost Facebook engagement for small businesses. http://www.socialmediaexaminer.com/boostfacebook-engagement-for-small-businesses/

Zarella, D., 2011. The science of social timing part 1: Social networks. Kissmetrics. https://blog.kissmetrics.com/science-of-socialtiming-1/ 